\title{
Disorder-induced metal-insulator transitions in three-dimensional topological insulators and superconductors
}

\author{
Shinsei Ryu ${ }^{1,2}$ and Kentaro Nomura ${ }^{3}$ \\ ${ }^{1}$ Department of Physics, University of Illinois, 1110 West Green St, Urbana IL 61801 \\ ${ }^{2}$ Condensed Matter Theory Laboratory, RIKEN, Saitama 351-0198, Japan \\ ${ }^{3}$ Correlated Electron Research Group (CERG), RIKEN-ASI, Wako 351-0198, Japan
}

(Dated: November 4, 2018)

\begin{abstract}
We discuss the effects of disorder in time-reversal invariant topological insulators and superconductors in three spatial dimensions. For three-dimensional topological insulator in symplectic (AII) symmetry class, the phase diagram in the presence of disorder and a mass term, which drives a transition between trivial and topological insulator phases, is computed numerically by the transfer matrix method. The numerics is supplemented by a field theory analysis (the large- $N_{f}$ expansion where $N_{f}$ is the number of valleys or Dirac cones), from which we obtain the correlation length exponent, and several anomalous dimensions at a non-trivial critical point separating a metallic phase and a Dirac semi-metal. A similar field theory approach is developed for disorder-driven transitions in symmetry class AIII, CI, and DIII. For these three symmetry classes, where topological superconductors are characterized by integer topological invariant, a complementary description is given in terms of the non-linear sigma model supplemented with a topological term which is a three-dimensional analogue of the Pruisken term in the integer quantum Hall effect.
\end{abstract}

\section{INTRODUCTION}

A topological phase is a gapped quantum state of matter with non-trivial quantum correlation (entanglement). Their key distinction from trivial states a band insulator, say, lies in the fact that they support stable gapless boundary modes when terminated by a boundary. A systematic classification of topological insulators and super conductors in all Altland-Zirnbauer symmetry classest and in all dimensions was obtained, revealing mod 2 and 8 periodicities in dimensionality and symmetry classes.2. It was found in any dimensions there exist topologically non-trivial insulators or superconductors for five out of ten Altland and Zirnbauer symmetry classes.

In three spatial dimensions, among the five symmetry classes is symplectic (spin-orbit) symmetry class (class AII) in which $\mathbb{Z}_{2}$ topological insulators can be realized.510 A number of materials with non-trivial $\mathbb{Z}_{2}$ topological number have been discovered, starting from Bismuth-Antimony alloys. (See, for a partial list of such materials, Refs. 11 14)

Topological superconductors that are possible in three dimensions are all time-reversal symmetric; they are realized in symmetry class CI (conserved SU(2) spin rotation symmetry), AIII (partially conserved SU(2) spin rotation symmetry), and DIII (no SU(2) spin rotation symmetry). In these symmetry classes topologically different gapped states are characterized in the bulk by an integral, as opposed to $\mathbb{Z}_{2}$, topological number $\nu$ defined in terms of the spectral projector in momentum space. In these topological superconductors, the Bogoliubov quasiparticles are fully gapped in the bulk by the (mean field) pairing gap, and the system is a thermal insulator. (When $z$-component of spin is a good quantum number as in symmetry class CI and AIII, spin transport can be discussed; topological superconductors in these classes are a spin insulator in the bulk.) On the other hand, when such three-dimensional (3d) topological superconductors are terminated by a $2 \mathrm{~d}$ surface, they can support gapless and stable Dirac (class AIII and CI) or Majorana (class DIII) surface modes, the multiplicity (number of Dirac or Majorana cones) of which is specified by the bulk topological number $\nu$. The fermionic quasiparticles in the B phase of ${ }^{3} \mathrm{He}$ (the BW state) realize an example of topological superconductor (superfluid) in class DIII. Singlet BCS pairing models on the diamond and cubic lattices that realize a topological superconductor in class CI have also been proposed 15,16

While topological insulators and superconductors are stable against weak perturbations, with strong perturbations, they can undergo a (continuous) quantum phase transition into a different phase. For example, if one can tune the chemical potential in the BW state, there is a transition between a trivial phase (strong pairing phase) and a topological phase (weak pairing phase). Likewise in the quantum Hall effect (QHE), strong enough disorder can also destroy the topological insulators and superconductors, and drive a system, through a continuous quantum phase transition, into a metal or other topological/trivial phases.

This paper is devoted to study such quantum phase transitions among topological (electrical, thermal or spin) insulators, trivial insulators, and a metal, in symmetry class AII, and in three Bogoliubov-de Gennes (BdG) symmetry classes CI, AIII and DIII. These quantum phase transitions can be induced by changing parameters that modify the band structure and wavefunctions, and also by introducing disorder; both of these perturbations are assumed to be preserving discrete symmetries defining these symmetry classes. 


\section{A. main results and outline of the paper}

a. symplectic (AII) symmetry class We start, in Sec. II by introducing a 3d Dirac-type Hamiltonian on the cubic lattice in symplectic (AII) symmetry class, which realizes a topological insulator, a trivial insulator, and a phase transition between these two. In Subsec. IIB, the phase diagram in the presence of a mass term and disorder is determined numerically by the transfer matrix method. (For earlier studies, see, for example, Refs. 17 20.) We observe that with disorder the phase boundary between topological and trivial phases is renormalized, and in particular the topological phase gets "enlarged". This means, as we pass through along a particular cut in the phase diagram, we can turn a trivial insulator into a topological one by increasing disorder strength ("topological Anderson insulator").

The numerical analysis is supplemented with a field theory analysis (the large- $N_{f}$ expansion of a GrossNeveu-type model where $N_{f}$ is the number of valleys or Dirac cones), in Subsec. II d, from which we obtain the correlation length exponent, several anomalous dimensions at non-trivial critical point, and dynamical exponent.

b. BdG symmetry classes AIII, DIII and CI In time-reversal symmetric superconducting symmetry classes (class AIII, DIII and CI), we will develop, in Sec. III, a similar large- $N_{f}$ field theory approach in terms of chiral Gross-Neveu- or Nambu-Jona-Lasinio-type models and their large- $N_{f}$ expansion.

While description in terms of these fermionic field theories are reliable for a region of the phase diagram where spin or thermal conductivity is relatively small (in particular near the clean fixed point separating trivial and topological superconductors), deep in a spin or thermal metallic phase, on the other hand, a complementary description in terms of the non-linear sigma model (NL $\sigma \mathrm{M})$ is possible [Subsec. IIID]. For these three symmetry classes, where topological superconductors are characterized by the integer topological invariant $\nu$, the NL $\sigma \mathrm{Ms}$ can be supplemented with a topological term which is of integer type (related to $\pi_{3}(G / H)=\mathbb{Z}$ of the NL $\sigma \mathrm{M}$ target space $G / H)$; it is a higher dimensional analogue of the Pruisken term in the QHE21 23. We will compute the theta angle in the topological term in terms of microscopic parameters (the fermion mass and the imaginary part of the fermion self-energy in the self-consistent Born approximation). While we do not have an analytical tool to analyze the effects of the topological term on the nature of Anderson metal-insulator transitions, we will speculate on possible RG flows in terms of diagonal spin or thermal conductivity and the theta parameter.

\section{DISORDER IN THREE-DIMENSIONAL TOPOLOGICAL INSULATOR IN SYMMETRY CLASS AII}

\section{A. Hamiltonian and symmetries}

Let us start by considering the following tight-binding Hamiltonian24 25:

$$
\begin{aligned}
H_{0}= & \sum_{\mathbf{x}} \sum_{k=1,2,3}\left[\frac{i t}{2} c_{\mathbf{x}+\mathbf{e}_{k}}^{\dagger} \alpha_{k} c_{\mathbf{x}}-\frac{r}{2} c_{\mathbf{x}+\mathbf{e}_{k}}^{\dagger} \beta c_{\mathbf{x}}+\text { h.c. }\right] \\
& +(m+3 r) \sum_{\mathbf{x}} c_{\mathbf{x}}^{\dagger} \beta c_{\mathbf{x}}
\end{aligned}
$$

where $c_{\mathbf{x}}^{\dagger}, c_{\mathbf{x}}$ represents the four-component fermion creation/annihilation operator defined on a site $\mathbf{x}$ on the $3 \mathrm{~d}$ cubic lattice spanned by three orthogonal unit vectors $\mathbf{e}_{k=x, y, z}$, and the $4 \times 4$ gamma matrices in the Dirac representation are given by

$$
\alpha_{k}=\left(\begin{array}{cc}
0 & \sigma_{k} \\
\sigma_{k} & 0
\end{array}\right), \beta=\left(\begin{array}{cc}
1 & 0 \\
0 & -1
\end{array}\right), \gamma_{5}=\left(\begin{array}{ll}
0 & 1 \\
1 & 0
\end{array}\right),
$$

with $\sigma_{k=1,2,3}$ being the standard Pauli matrices. In momentum space, the Hamiltonian is written as $H_{0}=$ $\sum_{\mathbf{k}} c_{\mathbf{k}}^{\dagger} \mathcal{H}_{0}(\mathbf{k}) c_{\mathbf{k}}$, where $\mathcal{H}_{0}(\mathbf{k})$ is given by

$$
\begin{aligned}
& \mathcal{H}_{0}(\mathbf{k})=\sum_{a=1}^{3} d_{a}(\mathbf{k}) \alpha_{a}+d_{4}(\mathbf{k}) \beta \\
& \text { with }\left\{\begin{array}{l}
d_{a}(\mathbf{k})=t \sin k_{a}, \quad(a=1,2,3), \\
d_{4}(\mathbf{k})=m+r \sum_{a=1}^{3}\left(1-\cos k_{a}\right),
\end{array}\right.
\end{aligned}
$$

where $\mathbf{k}=\left(k_{x}, k_{y}, k_{z}\right) \in[-\pi, \pi)^{3}$. For arbitrary values of the parameters $t, m, r \in \mathbb{R}$, the Hamiltonian is timereversal symmetric, $\left(i \sigma_{y}\right) \mathcal{H}_{0}^{*}(-\mathbf{k})\left(-i \sigma_{y}\right)=\mathcal{H}_{0}(\mathbf{k})$. In the following we fix the Wilson parameter $(r)$ and the hopping amplitude $(t)$ as $r=1$ and $t=2$.

As we change $m$ in the Hamiltonian, we can realize the trivial insulator, the $\mathbb{Z}_{2}$ topological insulator, and also a quantum critical point separating trivial and topological insulators:

- For $m<-6,-4<m<-2$ and $0<m, H_{0}$ realizes a trivial insulator whereas

- for $-6<m<-4$ and $-2<m<0, H_{0}$ realizes a topological insulator.

In the following we will mainly focus on the region near $m=0$.

We add, to $H_{0}$, the following disorder potential

$$
V=\sum_{\mathbf{x}} v_{\mathbf{x}} c_{\mathbf{x}}^{\dagger} c_{\mathbf{x}}
$$

which is time-reversal symmetric, and where the random variable $v_{\mathbf{x}}$ is distributed between $-W / 2$ and $W / 2$ according to the box-distribution. 
When we are close to a quantum critical point, say, at $m=0$, the tight binding Hamiltonian can be described in terms of its continuum limit, which takes the form of 3d massive Dirac Hamiltonian

$$
\mathcal{H}=\mathcal{H}_{0}+\mathcal{V}, \quad \mathcal{H}_{0}=-i \partial_{k} \alpha_{k}+m \beta,
$$

where the summation over repeated indices $k=1,2,3$ is implicit, and $\mathcal{V}$ represents a disorder potential. For any realization of disorder, the continuum Hamiltonian (5) satisfies time-reversal symmetry

$$
i \sigma_{y} \mathcal{H}^{*}\left(-i \sigma_{y}\right)=\mathcal{H} .
$$

The clean part of the Hamiltonian (5) $\mathcal{H}_{0}$ realizes, depending on the sign of the mass term $m \in \mathbb{R}$, a topologically trivial and non-trivial insulator in symmetry class AII, each of which is characterized by the vanishing and non-vanishing, respectively, of the $\mathbb{Z}_{2}$ invariant. When $m=0$ and in the absence of disorder, the spectrum is gapless whilst the dc conductivity is zero, $\sigma_{x x}=0$. This should be compared with the "unizersal" finite conductivity in the 2d Dirac Hamiltonian.26

\section{B. numerics}

c. transfer matrix method In this section, we will study numerically effects of disorder in the 3d Dirac model. Below, we use the transfer matrix to study the random Hamiltonian $H=H_{0}+V$. To this end, we write the "Dirac equation" for the single-particle wavefunction $\psi(\mathbf{x}, \sigma)=\psi(\mathbf{r}, \sigma)_{n}$ at a given energy $E$ in the following form:

$$
\begin{aligned}
\psi_{n+1}= & \frac{2}{1-t^{2}}\left(\beta+i t \alpha_{z}\right)\left[\mathcal{H}^{(n)}-E\right] \psi_{n} \\
& -\frac{1}{1-t^{2}}\left(\beta+i \alpha_{z}\right)\left(\beta-i \alpha_{z}\right) \psi_{n-1} .
\end{aligned}
$$

Here we decomposed the $3 \mathrm{~d}$ spatial coordinates $\mathbf{x}$ in terms of its $x$ - and $y$-components $\mathbf{r}$ and its $z$-component $n, \mathbf{x}=(\mathbf{r}, n)$, and $\mathcal{H}^{(n)}$ is the Hamiltonian for the $n$th cross section. As shown in Appendix A, this can be represented as

$$
\begin{aligned}
& \left(\begin{array}{c}
\psi_{n+1} \\
\psi_{n}
\end{array}\right)=M^{(n)}\left(\begin{array}{c}
\psi_{n} \\
\psi_{n-1}
\end{array}\right), \quad M^{(n)}=\left(\begin{array}{cc}
h^{(n)} & v \\
1 & 0
\end{array}\right), \\
& \text { where } h^{(n)} \equiv \frac{-2}{t^{2}-1}\left(\beta+i t \alpha_{z}\right)\left[\mathcal{H}^{(n)}-E\right] .
\end{aligned}
$$

The size of each slice with fixed $n$ is $L \times L$, so that $M^{(n)}$ is a $4 L \times 4 L$ matrix. We will denote the length of the $z$-direction by $N$.

We study numerically the dependence of the smallest Lyapunov exponent of the transfer matrix $M$, as a function of the width $L^{2}$ of the quasi-one dimensional system. Lyapunov exponents are self-averaging random variables for an infinitely long quasi-one dimensional system, $N \rightarrow \infty$.
The eigenvalues of the Hermitian matrix $M^{\dagger} M$ are written as $\exp \left( \pm 2 X_{j}\right)$ with $0<X_{1}<X_{2}<\ldots<X_{4 L}$. The decay length $\xi_{L}$ is then given by

$$
\xi_{L}=\lim _{N \rightarrow \infty} \frac{1}{N X_{1}} .
$$

The decay length $\xi_{L}$ is a finite and self-averaging length scale that controls the exponential decay of the Landauer conductance for any fixed width $L$ of the infinitely long quasi-one dimensional system. It is of course impossible to study infinitely long quasi-one dimensional lattice models numerically and we shall approximate $\xi_{L}$ with $\xi_{L, N}$ obtained from the Lyapunov exponents of a finite but long quasi-one dimensional network model made of $N$ slices. In our numerics we have set $N=1 \times 10^{5} \sim 5 \times 10^{6}$.

As shown by MacKinnon and Kramer,27 criticality can be accessed from the dependence of the normalized decay length

$$
\Lambda_{L}:=\xi_{L} / L
$$

on the width $L$ of the quasi-one dimensional system. As usual, $\Lambda_{L}$ is required to be written as $\Lambda_{L}=f(L / \xi)$ on the basis of the single-parameter scaling hypothesis, where $\xi$ is the (3d) localization length. At the critical point the localization length diverges according to the power low $\xi \sim\left|x-x_{c}\right|^{-1 / \nu}$ upon tuning of a single microscopic parameter $x$ (disorder strength $W$ or mass $m$ in the following).

The numerically computed normalized decay length is shown for $m=0$ and for different disorder strength in Fig. 1. The plots for different values of $L(=6,8,10$, and 12) cross at a single point $W \sim 3.9$, signaling an insulator-to-metal transition. The inset shows $\Lambda_{L}$ as a function of $L / \xi$ with the help of the one-parameter scaling ansatz. In a metallic region $\Lambda_{L}$ fits well with a typical metal-insulator transition behavior, while in the insulating phase $L$-dependence gets severer at weak disorder. This is so since in the insulating phase, the presence of the band gap would complicate the scaling analysis. The fact that in the insulating side the data are not well fit by the scaling curve also makes the unambiguous determination of the critical exponent difficult. A similar problem was observed in the metal-ipsulator transition in the $2 \mathrm{~d}$ quantum spin Hall systems.28 30 A special effort on our $3 \mathrm{~d}$ model will be made elsewhere.

d. structure of the phase diagram In Fig. 2, the numerically determined phase diagram is shown. Observe that as we increase disorder the phase boundary separating trivial and topological insulators gets shifted toward the large mass region, i.e., the trivial insulator region becomes topologically non-trivial as disorder strength increases. This disorder-induced topological insulating phase has been found first in tyo dimension 3134 and recently in three dimensions 1920 , and thereby referred to as "topological Anderson insulator". (However, one should note that the determination of the phase boundary between the two insulating phases is rather difficult 


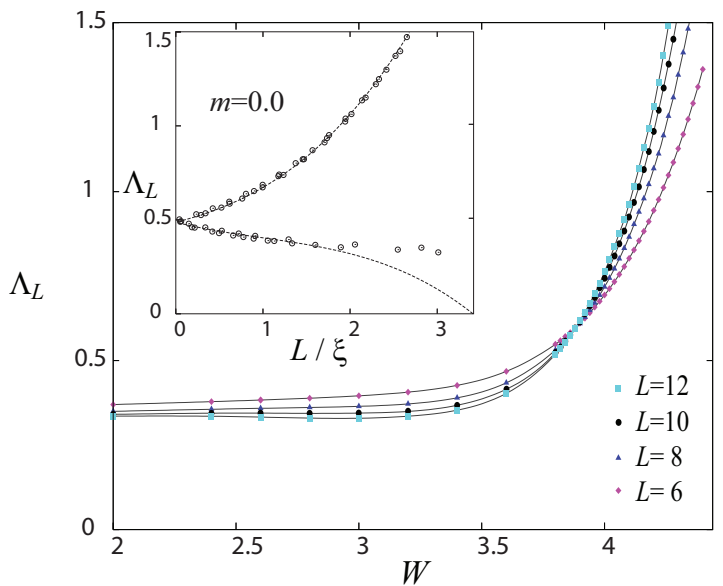

FIG. 1. Normalized decay length $\Lambda_{L}=\Lambda / L$ as a function of disorder strength $W$ for $m=0.0$. Inset: A fit of the data shown in the main panel with the help of the one-parameter scaling ansatz in the vicinity of the critical point.

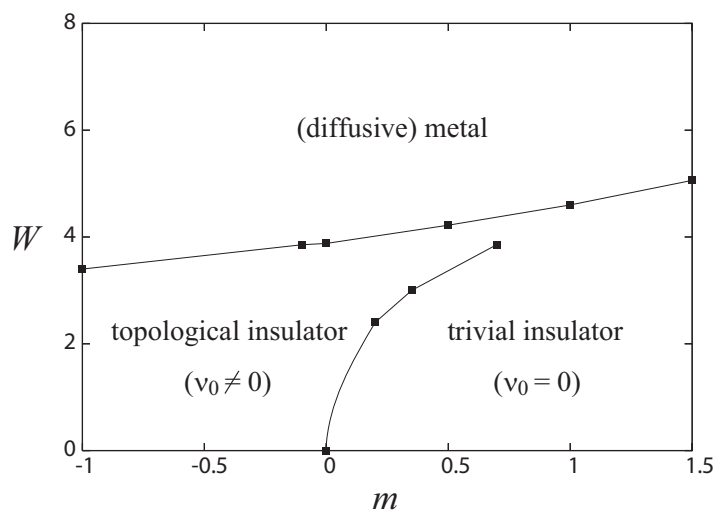

FIG. 2. The numerically obtained phase diagram in the $(W, m)$ plane. Lines are guide for eyes.

numerically since in an insulator the $L$ dependence of the decay length is intrinsically small.) While it is not depicted here, in the strong disorder limit, we expect there should be another phase boundary separating the metallic phase and an insulator.

\section{C. field theory}

We now study the metal insulator transition from field theory point of view. Our starting point is the continuum limit of the lattice tight-binding model, Eq. (5),

$$
\mathcal{H}=\mathcal{H}_{0}+\mathcal{V}, \quad \mathcal{H}_{0}=-i \partial_{k} \alpha_{k}+m \beta .
$$

With time reversal symmetry, the disorder potential $\mathcal{V}$ can be expanded in terms of $\sigma_{x, z} \tau_{y}, \sigma_{0} \tau_{0, x, z}$, and $\sigma_{y} \tau_{y}$, and hence parameterized by six real parameters characterizing disorder. Below, we assume, for simplicity, dis- order potentials are distributed according to a Gaussian distribution, and their variances are all equal,

$$
P(V) \propto \exp \left[-\frac{1}{2 g} \operatorname{tr}_{4} \mathcal{V} \mathcal{V}^{\dagger}\right]
$$

where $g$ is a parameter that characterizes the strength of disorder.

\section{1. fermionic replica method}

We will use the fermionic replica method for quenched disorder averaging. In the fermionic replica method, the discrete symmetry of the problem, time-reversal symmetry, is implemented as a continuous symmetry: invariance under $\mathrm{O}\left(2 N_{r}\right) \times \mathrm{O}\left(2 N_{r}\right)$ rotations in the replica space where $N_{r}$ is the number of replicas.

In order to study Anderson localization physics, we are after the properties of the single particle retarded $(\mathrm{R})$ and advanced (A) Green's function, $\left( \pm i 0^{+}-\mathcal{H}\right)^{-1}$, and in particular, the product thereof, where $0^{+}>0$ is a small imaginary part of the energy needed to regularize poles. For a product of the retarded $(\mathrm{R})$ and advanced (A) Greens functions, $\propto\left(i 0^{+}-\mathcal{H}\right)^{-1}\left(i 0^{+}+\mathcal{H}\right)^{-1}$, its generating function can be expressed as a fermionic functional integral, $Z=\int \mathcal{D}[\bar{\Psi}, \Psi] \exp \left(-\int d^{3} x \mathcal{L}\right)$, where

$$
\mathcal{L}=\bar{\Psi}\left(i \mathcal{H}+0^{+} \Lambda\right) \Psi .
$$

Here, the total number of components of the fermion field $\bar{\Psi}, \Psi$ is

$$
\begin{aligned}
& (\text { Dirac }) \otimes(\mathrm{TR}) \otimes(\mathrm{R} / \mathrm{A}) \otimes(\text { replica }) \\
& \quad=\sigma \otimes \tau \otimes(\mathrm{R} / \mathrm{A}) \otimes(\text { replica })=16 N_{r}
\end{aligned}
$$

where $\Lambda$ is a diagonal matrix which is \pm 1 for the retarded/advanced sector, respectively. The fermionic field $\Psi$ incorporates the time-reversal symmetry grading, and can be written as

$$
\bar{\Psi}=\frac{1}{\sqrt{2}}\left(\bar{\chi},-\chi^{T} i \sigma_{y}\right)_{\tau}, \Psi=\frac{1}{\sqrt{2}}\left(\begin{array}{c}
\chi \\
-i \sigma_{y} \bar{\chi}^{T}
\end{array}\right)_{\tau},
$$

where $\chi, \bar{\chi}$ are two-independent fermionic fields (we have suppressed the replica indices.); the subscript $(\cdots)_{\tau}$ means the block structure displayed in Eq. (15) represents the time-reversal symmetry grading. It will prove convenient to rewrite Eq. (15) in the spin grading in which the block structure displays subspaces with $\sigma= \pm$ :

$$
\bar{\Psi}=\frac{1}{\sqrt{2}}\left(\kappa^{T} \tau_{z y},-\gamma^{T} \tau_{z y}\right)_{\sigma}, \quad \Psi=\frac{1}{\sqrt{2}}\left(\begin{array}{c}
-i \tau_{z y} \gamma \\
-i \tau_{z y} \kappa
\end{array}\right)_{\sigma},
$$

where

$$
\begin{aligned}
& -\kappa^{T} \tau_{y z}:=\left(\bar{\chi}_{\uparrow}, \chi_{\downarrow}^{T}\right), \quad \gamma^{T} \tau_{y z}:=\left(\bar{\chi}_{\downarrow},-\chi_{\uparrow}^{T}\right), \\
& \tau_{z y}=\left(\tau_{z}-\tau_{y}\right) / \sqrt{2} .
\end{aligned}
$$


With these, the action can be written correspondingly as

$$
\begin{aligned}
& \bar{\Psi}(i \mathcal{H}+\eta \Lambda) \Psi \\
= & \frac{-i}{2}\left(-\kappa^{T}, \gamma^{T}\right)_{\sigma}(i \mathcal{H}+\eta \Lambda)\left(\begin{array}{c}
\gamma \\
\kappa
\end{array}\right)_{\sigma} \\
= & \frac{-i}{2}\left(\gamma^{T}, \kappa^{T}\right)_{\sigma} i \sigma_{y}(i \mathcal{H}+\eta \Lambda)\left(\begin{array}{c}
\gamma \\
\kappa
\end{array}\right)_{\sigma} .
\end{aligned}
$$

By introducing four component spinors by

$$
\psi=\frac{1}{\sqrt{2}}\left(\begin{array}{c}
\gamma \\
\kappa
\end{array}\right)_{\sigma}, \quad \bar{\psi}=\psi^{T} C^{-1}=\psi^{T} i \sigma_{y},
$$

we end up with the functional integral $Z=$ $\int \mathcal{D}[\bar{\psi}, \psi] \exp \left(-\int d^{3} x \mathcal{L}\right)$ with the Lagrangian

$$
\mathcal{L}=\sum_{a, b=1}^{4 N_{r}} \bar{\psi}_{a}\left(i \mathcal{H} \delta_{a b}+0^{+} \Lambda_{a b}\right) \psi_{b}
$$

When $0^{+}=0$, the action is invariant under $\mathrm{O}\left(4 N_{r}\right)$ rotations, $\kappa \rightarrow U \kappa, \gamma \rightarrow U \gamma, U \in \mathrm{O}\left(4 N_{r}\right)$. In the metallic phase where a small imaginary part in the self-energy is generated spontaneously, this symmetry is broken down to $\mathrm{O}\left(2 N_{r}\right) \times \mathrm{O}\left(2 N_{r}\right)$. The resulting Nambu-Goldstone mode is the diffuson in the metallic phase.

We can now perform the disorder averaging according to $P(V)$, resulting in the generating functional $Z=$ $\int \mathcal{D}[\bar{\psi}, \psi] \exp \left(-\int d^{3} x \mathcal{L}\right)$ with the Lagrangian

$$
\mathcal{L}=\sum_{a, b=1}^{4 N_{r}} \bar{\psi}_{a}\left(i \mathcal{H}_{0} \delta_{a b}+0^{+} \Lambda_{a b}\right) \psi_{b}-\frac{g}{2} \sum_{a, b} \bar{\psi}_{b} \psi_{a} \bar{\psi}_{a} \psi_{b} .
$$

When $\mathrm{g}^{+}$is zero, this is a $3 \mathrm{~d}$ version of the Gross-Neveu model 35 with $\mathrm{O}\left(4 N_{r}\right)$ internal and $\mathrm{O}\left(N_{f}\right)$ flavor symmetries.

\section{2. saddle point}

The disorder-induced four-fermion "interaction" can be decoupled by an auxiliary matrix field $W_{a b}$ leading to $Z=\int \mathcal{D}[\bar{\psi}, \psi] \int \mathcal{D}[W] \exp \left(-\int d^{3} x \mathcal{L}\right)$,

$$
\begin{aligned}
\mathcal{L}= & \sum_{\iota=1}^{N_{f}} \sum_{a, b=1}^{4 N_{r}} \bar{\psi}_{\iota a}\left(i \mathcal{H}_{0} \delta_{a b}+0^{+} \Lambda_{a b}+W_{a b}\right) \psi_{\iota b} \\
& +\frac{1}{2 g} \operatorname{tr}_{4 N_{r}}\left[W W^{T}\right] .
\end{aligned}
$$

Here, we have introduced $N_{f}$ flavors of fermion fields $\psi_{\iota a}\left(\iota=1, \ldots, N_{f}\right)$. Equation (22) reduces to the situation we have been discussing when $N_{f}=1$, whereas $N_{f}>1$ corresponds to the case with $N_{f}$ "valleys". The replicated Lagrangian (22) can be derived from a model similar to (11) once we assume all the flavors are coupled equally by a disorder potential. (See Appendix B.) We

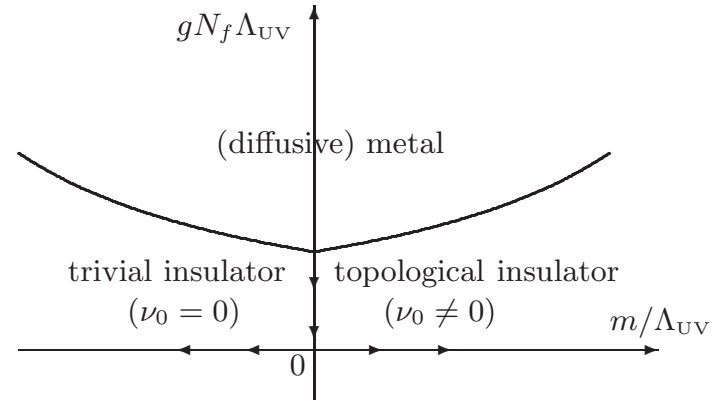

FIG. 3. The mean field (self-consistent Born) phase diagram near the Dirac point. Arrows indicate the renormalization group flow.

will discuss the four-fermion "interaction" term in terms of the large $N_{f}$ expansion below.

As a first step, we now look for a spatially homogeneous, replica symmetric saddle point solution, by setting

$$
W_{a b}=\eta \Lambda_{a b}
$$

This reduces the symmetry $\mathrm{O}\left(4 N_{r}\right) \rightarrow \mathrm{O}\left(2 N_{r}\right) \times \mathrm{O}\left(2 N_{r}\right)$. The self-consistency conditions for $\eta$ is given by

$$
\eta \int^{\Lambda_{\mathrm{UV}}} \frac{d^{3} k}{(2 \pi)^{3}} \frac{1}{k^{2}+m^{2}+\eta^{2}}-\frac{\eta}{4 N_{f} g}=0
$$

where we have introduced the ultra-violet cutoff $\Lambda_{\mathrm{Uv}}$. Anticipating spontaneous breaking of $\mathrm{O}\left(4 N_{r}\right)$ symmetry, we have set $0^{+}=0$, as its sole role is to select the pattern of the residual symmetry, $\mathrm{O}\left(2 N_{r}\right) \times \mathrm{O}\left(2 N_{r}\right)$.

The resulting mean-field (or self-consistent Born) phase diagram is given in Fig. 3. We identify the phase with $\eta \neq 0$ (the ordered phase with spontaneous $\mathrm{O}\left(4 N_{r}\right)$ symmetry breaking) as a (diffusive) metallic phase. This metallic phase appears because disorder creates states that fill the pseudo band gap of the Dirac fermions. On the other hand, there are two, trivial and topological, insulating phases with $\eta=0$ which are separated by a phase boundary which runs vertically at $m=0$.

In the lattice Dirac model (11), a unitary transformation $c_{\mathbf{x}} \rightarrow i \gamma_{5} c_{\mathbf{x}}, c_{\mathbf{x}}^{\dagger} \rightarrow-c_{\mathbf{x}}^{\dagger} i \gamma_{5}$ relates two Hamiltonians, one with the parameter $(m, r)$ and the other with $(-m,-r)$; i.e., Hamiltonians with the mass $m$ and $-m$ are not unitary equivalent if $r$ is held fixed. On the other hand, the continuum Dirac model (11) and its replicated counterpart (21) are invariant under a $\mathbb{Z}_{2}$ transformation $\psi_{a} \rightarrow \gamma_{5} \psi_{a}, \psi_{a} \rightarrow \bar{\psi}_{a} \gamma_{5}, m \rightarrow-m$. For this reason, while the numerical phase diagram Fig. (2) is asymmetric under $m \leftrightarrow-m$, the mean field phase diagram Fig. 3 is symmetric under $m \leftrightarrow-m$; the "topological Anderson insulator" phase is not possible in the continuum model with linear dispersion. 


\section{3. $1 / N_{f}$-expansion}

Just at the Dirac point, the disorder, which appears as a four fermion interaction in the generating functional, is irrelevant, whereas the mass term is relevant, from the power-counting. The transition between trivial and topological insulators is then described by the clean Dirac point. We will now turn our attention to the non-trivial fixed point at $(m, g)=\left(0, g_{c}\right)$, where $g_{c} \neq 0$ is the nontrivial solution to the saddle point equation Eq. (24). To include $1 / N_{f}$ corrections systematically, we consider the Lagrangian

$$
\begin{aligned}
\mathcal{L}= & Z_{\psi} \bar{\psi}_{\iota a}\left(\partial_{k} \gamma_{k} \delta_{a b}+\Sigma \Lambda_{a b}\right) \psi_{\iota b} \\
& +\frac{g}{\sqrt{N_{f}}} Z_{\psi} Z_{W}^{1 / 2} \bar{\psi}_{\iota a} W_{a b} \psi_{\iota b}+\frac{1}{2} Z_{W} W_{a b} W_{a b},
\end{aligned}
$$

where repeated flavor and replica indices are implicitly summed, and we have introduced Euclidean gamma matrices $\gamma_{k}=\alpha_{k}, g$ is the bare coupling and $Z_{\psi}, Z_{W}$ are wavefunction renormalization constants; we have also rescaled the bosonic field $W_{a b}$ properly. These renormalization constants must be adjusted at each order in $1 / N_{f}$ to keep vertex functions (in particular, the fermion propagator, the fermion-boson vertex, and the boson propagator) finite. (See, for example, Refs. 36 39.)

To leading order in $1 / N_{f}$, as we have seen in the saddle point analysis, the expectation value of $W(=\eta)$ is spontaneously generated if $g>g_{c}$ : the self-consistency equation is

$$
\eta \int^{\Lambda_{\mathrm{UV}}} \frac{d^{3} k}{(2 \pi)^{3}} \frac{1}{k^{2}+\eta^{2}}-\frac{\eta}{N_{f} D_{\gamma} g}=0,
$$

where $D_{\gamma}(=4)$ is the dimensionality of the Euclidean gamma matrices. The expectation value $\eta$ is a physical observable which sets a scale, and hence independent of the UV cutoff. We will go to the non-trivial branch $g>g_{c}$ of the saddle point equation (26) for the most part of our analysis below. This equation relates the bare coupling constant $g$ to the UV cutoff and a physical scale $\eta$, and can be considered as a renormalization condition.

The fermion propagator to leading order is given by

$$
\begin{aligned}
\left\langle\psi_{\iota a}(k) \bar{\psi}_{\kappa b}(k)\right\rangle & =\delta_{\iota \kappa} \mathcal{G}_{0, a b}(k), \\
\mathcal{G}_{0}^{-1}(k) & =2 Z_{\psi}\left(i k_{i} \gamma_{i}+\Sigma \Lambda_{a b}\right),
\end{aligned}
$$

where we can choose $Z_{\psi}=1$ and $\Sigma=\eta$ to this order. The factor of 2 reflects the Majorana nature of the fermion fields.

The boson two point function to leading order can be computed by summing over fermion bubble diagrams (see Fig. (4),

$$
\left\langle W_{a b}(q) W_{c d}\left(-q^{\prime}\right)\right\rangle=\frac{8}{Z_{W} g^{2} D_{\gamma}} \mathcal{D}(q) \delta_{q, q^{\prime}}\left(\delta_{c b} \delta_{d a}+\delta_{c a} \delta_{d b}\right),
$$

(a)

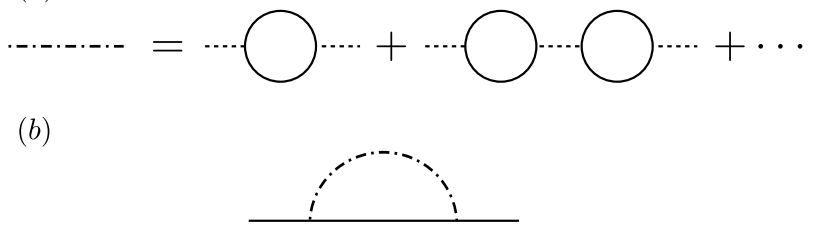

FIG. 4. Some Feynman diagrams appearing in the $1 / N_{f}$ expansion. (a): summing fermion bubble diagrams [Eq. (28)]. (b): the $1 / N_{f}$ expansion correction to the fermion self-energy [Eq. (32)].

where $a, b, c, d=1, \ldots, 4 N_{r}, \delta_{q, q^{\prime}}:=(2 \pi)^{3} \delta\left(q-q^{\prime}\right)$, and we have introduced

$$
\mathcal{D}(q):=\frac{8 \pi|q|}{\left(q^{2}+4 \Sigma^{2}\right) \operatorname{atan}(|q| / 2 \Sigma)} .
$$

In the UV limit (i.e., at the critical point $g=g_{c}$ ),

$$
\mathcal{D}(q) \rightarrow 4 /|q|,
$$

and hence, by choosing the renormalization constant as $Z_{W} g^{2}=\Sigma^{-1}$,

$$
\left\langle W_{a b}(q) W_{c d}\left(-q^{\prime}\right)\right\rangle=\frac{32}{D_{\gamma}|q|} \delta_{q, q^{\prime}}\left(\delta_{c b} \delta_{d a}+\delta_{c a} \delta_{d b}\right) .
$$

The critical exponent $\nu$ is given, to leading order in $1 / N_{f}$, by $\nu=1 /(d-2)$ where $d=3$. We now compute next to leading order (the order $1 / N_{f}$ ) correction to the critical exponent $\nu$. First, we compute the wavefunction renormalization and mass renormalization to nextto-leading order. The fermion propagator with $1 / N_{f}$ corrections is given by

$$
\begin{aligned}
\mathcal{G}^{-1}(k)= & 2 Z_{\psi}\left\{i k_{i} \gamma_{i} \delta_{a b}\left[1+\frac{16\left(4 N_{r}+1\right)}{3 \pi^{2} N_{f} D_{\gamma}} \ln \frac{\Lambda_{\mathrm{UV}}}{|\eta|}\right]\right. \\
& \left.+\Sigma \Lambda_{a b}\left[1-\frac{16}{\pi^{2} N_{f} D_{\gamma}} \ln \frac{\Lambda_{\mathrm{UV}}}{|\eta|}\right]\right\} .
\end{aligned}
$$

From this, we determine the counter terms as

$$
\begin{aligned}
& Z_{\psi}=1-\frac{16\left(4 N_{r}+1\right)}{3 \pi^{2} N_{f} D_{\gamma}} \ln \frac{\Lambda_{\mathrm{UV}}}{|\eta|}, \\
& \Sigma=\eta\left[1+\alpha \ln \frac{\Lambda_{\mathrm{UV}}}{|\eta|}\right]=\eta\left(\frac{\eta}{\Lambda_{\mathrm{UV}}}\right)^{-\alpha} \\
& \text { with } \quad \alpha=\frac{64\left(N_{r}+1\right)}{3 \pi^{2} N_{f} D_{\gamma}} .
\end{aligned}
$$

By definition, the critical exponent $\nu$ is determined from the divergence of the correction length $\xi$ as a function of the distance from the critical point,

$$
t:=g^{2}\left(g_{c}^{-2}-g^{-2}\right) \propto \xi^{-1 / \nu} .
$$


Note also that $\xi$ is inversely proportional to $\eta$. On the other hand, to this order, $t \propto \Sigma^{d-2}$. Combining these,

$$
\begin{gathered}
t \propto \eta^{(d-2)(1-\alpha)}=\xi^{-(d-2)(1-\alpha)} \\
\Rightarrow \quad \nu=\frac{1}{(d-2)(1-\alpha)}=\frac{(1+\alpha)}{(d-2)} .
\end{gathered}
$$

In particular, when $d=3, N_{f}=1, D_{\gamma}=4$, and in the replica limit $N_{r} \rightarrow 0$,

$$
\nu=1+\frac{16}{3 \pi^{2}} \simeq 1.54 \text {. }
$$

The result should be compared with the critical exponent at the conventional Anderson transition in symplectic (AII) symmetry class, which is estimated numerically as $\nu \sim 1.3-1.4$. (See Ref. 40 and references therein.)

a. dynamical exponent So far we have been interested in static properties. Some dynamical properties can also be computed within the large- $N_{f}$ expansion. To this end, let us start from the imaginary-time action

$$
S=\int d \tau d^{d} x \sum_{\iota, \kappa=1}^{N_{f}} \psi_{\iota}^{\dagger}\left[\left(\partial_{\tau}+\mathcal{H}_{0}\right) \delta_{\iota \kappa}+\mathcal{V}_{\iota \kappa}\right] \psi_{\kappa}
$$

Within the imaginary-time action, electron-electron interaction terms such as $(\lambda / 2) \int d \tau d^{d} x\left(\sum_{\iota=1}^{N_{f}} \bar{\psi}_{\iota} \psi_{\iota}\right)^{2}$ can be added. As before, disorder can be averaged by the fermionic replica method, and then the subsequent, disorder-induced "interaction" term can be decoupled in terms of the Hubbard-Stratonovich field $W\left(\tau, \tau^{\prime}, x\right)$ as

$$
\begin{aligned}
S= & \int d \tau d^{d} x \sum_{a=1}^{N_{r}} \psi_{a}^{\dagger}\left(\partial_{\tau}+\mathcal{H}_{0}\right) \psi_{a} \\
& +i \int d^{d} x \int d \tau d \tau^{\prime} \sum_{a, b} \psi_{a}^{\dagger}(\tau) W_{a b}\left(\tau, \tau^{\prime}\right) \psi_{b}\left(\tau^{\prime}\right) \\
& +\frac{1}{2 g} \int d^{d} x \int d \tau d \tau^{\prime} \sum_{a b} W_{a b}\left(\tau, \tau^{\prime}, x\right) W_{b a}\left(\tau^{\prime}, \tau, x\right) .
\end{aligned}
$$

This action can be studied by the saddle point method. We assume the saddle point is spatially homogeneous and replica symmetric,

$$
W_{a b}\left(\tau, \tau^{\prime}, x\right)=X\left(\tau, \tau^{\prime}\right) \delta_{a b},
$$

and $X\left(\tau, \tau^{\prime}\right)$ in frequency space is diagonal

$$
X\left(i \omega_{n}, i \omega_{n^{\prime}}\right)=\delta\left(\omega_{n}-\omega_{n^{\prime}}\right) X\left(i \omega_{n}\right)
$$

The electron Green function is given by, at the disorder-dominated critical point,

$$
\begin{aligned}
\mathcal{G}^{-1}\left(\omega_{n}, k\right) & =-i \omega_{n}+\mathcal{H}_{0}(k)+i X\left(i \omega_{n}\right) \\
& =\mathcal{H}_{0}(k)-i C_{d}\left|\omega_{n}\right|^{\frac{1}{d-1}} \operatorname{sgn} \omega_{n}
\end{aligned}
$$

where $C_{d}$ is a dimensionful numerical constant, $C_{d}=$ $\{[2|\sin (\pi d / 2)|] /[\pi(d-2)]\}^{1 /(d-1)} \Lambda_{\mathrm{UV}}^{(d-2) /(d-1)}$. From this, the dynamical exponent is

$$
z=d-1 \text {. }
$$

The Green's function (41) should be contrasted with the non-relativistic case where the disorder induced imaginary part of the self-energy within the self-consistent Born approximation is given by

$$
i X\left(i \omega_{n}\right)=-\frac{i}{2 \tau} \operatorname{sgn} \omega_{n}
$$

where $\tau=1 /\left[2 \pi \rho\left(\varepsilon_{F}\right) g\right]$ is the elastic scattering time with $\rho\left(\varepsilon_{F}\right)$ being the density of states at the Fermi energy $\varepsilon_{F} .41$

\section{DISORDER IN THREE-DIMENSIONAL TOPOLOGICAL SUPERCONDUCTORS}

The Bogoliubov-de Gennes (BdG) Hamiltonian describing the dynamics of fermionic quasiparticles in the superconducting (superfluid) phase realizes, depending on its symmetry properties, six out of ten Altland and Zirnbauer symmetry classes. They can be summarized, in terms of how badly $\mathrm{SU}(2)$ spin rotation symmetry is broken, as follows: A family (an ensemble) of BdG Hamiltonians with completely broken spin rotation symmetry is called symmetry class D (no TRS) or DIII (with TRS). When one component (z-component, say) of spin is conserved, symmetry class A (no TRS) and AIII (with TRS) are realized.42 43 Finally, when full $\mathrm{SU}(2)$ symmetry is preserved, class C (no TRS) and CI (with TRS) are realized.

It is worth mentioning that historically symmetry class A and AIII have been discussed mainly in the context of electronic systems (non BdG systems): In the original paper by Altland and Zirnbauer, class D, DIII, C, and ff are called "BdG classes", but not class A and AIII. 44 Symmetry class A, or unitary class, appears quite generally for electron systems which lack with TRS, and also with any other discrete symmetries. Symmetry class AIII describes an electronic system with broken TRS and with sublattice symmetry; i.e., tight-binding Hamiltonians with bipartite hopping elements only. While sublattice symmetry implies an invariance of the single-particle energy spectrum under reflection about zero energy, this is not related to PHS of BdG systems when a class AIII system is interpreted as describing BdG quasiparticles. Below, when we speak about symmetry class AIII, we will focus on its superconducting interpretation, since sublattice symmetry in electronic systems requires fine-tuning, while class AIII in BdG systems arises more naturally.

In three spatial dimensions, there are three symmetry classes, out of ten Altland and Zirnbauer symmetry classes, for which non-trivial topological phases characterized by an integer topological invariant exist. These are symmetry class CI, AIII and DIII. BdG Hamiltonians in these classes have two discrete symmetries, TRS and PHS of different kind and as a result they anticommute with a unitary matrix. For this reason, topological phases in these symmetry classes are called chiral topological superconductors in Ref. 16. 
In this section, we will discuss effects of disorder in these 3d topological superconductors. For the most part of this section, we will mainly focus on symmetry class AIII. The formalism for classes DIII and CI are largely parallel to class AIII, and will be discussed in Appendix.

\section{A. more descriptions of models}

b. BdG Hamiltonian The dynamics of quasiparticles deep inside a superconducting phase is described by a BdG Hamiltonian. On a lattice with $N$ sites, which is convenient for the purpose of discussing discrete symmetries of various kind, it can be written as follows:

$$
H=\frac{1}{2}\left(c^{\dagger}, c\right) \mathcal{H}_{4}\left(\begin{array}{c}
c \\
c^{\dagger}
\end{array}\right), \mathcal{H}_{4}=\left(\begin{array}{cc}
\Xi & \Delta \\
\Delta^{\dagger} & -\Xi^{T}
\end{array}\right),
$$

where $\mathcal{H}_{4}$ is a $4 N \times 4 N$ matrix and $\boldsymbol{c}=\left(\boldsymbol{c}_{\uparrow}, \boldsymbol{c}_{\downarrow}\right)$. $\quad[\boldsymbol{c}$ and $\boldsymbol{c}^{\dagger}$ can be either column or row vector depending on the context.] Because of $\Xi=\Xi^{\dagger}$ (hermiticity) and $\Delta=-\Delta^{T}$ (Fermi statistics), the BdG Hamiltonian (44) satisfies PHS

$$
[\mathrm{PHS}]: \mathcal{H}_{4}=-t_{x} \mathcal{H}_{4}^{T} t_{x}
$$

where the Pauli matrix $t_{x, y, z}$ is acting on the particlehole grading. In addition to PHS, depending on physical systems, TRS

$$
[\mathrm{TRS}]: \mathcal{H}_{4}=i s_{y} \mathcal{H}_{4}^{T}\left(-i s_{y}\right),
$$

and rotation symmetry for each spin rotation axis

$$
\begin{aligned}
& {[\mathrm{SU}(2) \text { symmetry }(a=x, y, z)]:} \\
& \quad\left[\mathcal{H}_{4}, J_{a}\right]=0, \quad J_{a}:=\left(\begin{array}{cc}
s_{a} & 0 \\
0 & -s_{a}^{T}
\end{array}\right),
\end{aligned}
$$

can be satisfied, where $s_{x, y, z}$ is the Pauli matrix acting on spin indices. Depending on their symmetry properties, BdG Hamiltonians (44) are classified into sub classes D, DIII, A, AIII, C, and CI.

\section{1. class DIII}

When both TRS and PHS are preserved in the BdG Hamiltonian, the relevant symmetry class is symmetry class DIII. Combining TRS and PHS, one can see that a member of class DIII anticommutes with the unitary matrix $t_{x} \otimes s_{y}$,

$$
\mathcal{H}_{4}=-t_{x} \otimes s_{y} \mathcal{H}_{4} t_{x} \otimes s_{y} .
$$

In this sense, class DIII Hamiltonians have a chiral structure. It is sometimes convenient to take a basis in which the chiral transformation, which is $t_{x} \otimes s_{y}$ in the present basis, is diagonal. In one of such bases, a class DIII Hamiltonian takes on the form

$$
\mathcal{H}_{4}=\left(\begin{array}{cc}
0 & D \\
D^{\dagger} & 0
\end{array}\right), \quad D=-D^{T}
$$

A canonical example of the class DHL togical superconductor is the $\mathrm{B}$ phase of ${ }^{3} \mathrm{He}$, 25.45 47 which is described, in momentum space, by the following BdG Hamiltonian:

$$
H=\frac{1}{2} \int d^{3} k \Psi^{\dagger}(\mathbf{k}) \mathcal{H}(\mathbf{k}) \Psi(\mathbf{k}),
$$

where $\Psi^{\dagger}(\mathbf{k})=\left(c_{\uparrow, \mathbf{k}}^{\dagger}, c_{\downarrow, \mathbf{k}}^{\dagger}, c_{\uparrow,-\mathbf{k}}, c_{\downarrow,-\mathbf{k}}\right)$ is the Nambu spinor composed of fermionic creation/annihilation operators $\left(c_{s, \mathbf{k}}^{\dagger} / c_{s, \mathbf{k}}\right)$ of a ${ }^{3} \mathrm{He}$ atom with spin $s$ and momentum $\mathbf{k}$, and the kernel takes the following form:

$$
\mathcal{H}(\mathbf{k})=\left(\begin{array}{cc}
\xi(\mathbf{k}) & \Delta(\mathbf{k}) \\
\Delta^{\dagger}(\mathbf{k}) & -\xi(-\mathbf{k})
\end{array}\right)
$$

The matrix elements are given by

$$
\begin{aligned}
& \xi(\mathbf{k})=k^{2} /(2 m)-\mu, \\
& \Delta(\mathbf{k})=\boldsymbol{d}(\mathbf{k}) \cdot \boldsymbol{s}\left(i s_{y}\right), \quad \boldsymbol{d}(\mathbf{k})=|\Delta| \mathbf{k},
\end{aligned}
$$

where $m$ is the mass of a ${ }^{3} \mathrm{He}$ atom, $\mu$ is the chemical potential, and $|\Delta|$ is the amplitude of the pair potential. With the $\boldsymbol{d}$-vector pointing parallel to momentum, there is an isotropic gap everywhere on the $3 \mathrm{~d}$ fermi surface. The critical point at $\mu=0$ separates topologically trivial $(\mu<0)$ and non-trivial $(\mu>0)$ phases, which are characterized by the topological invariant $\nu=0$ and $\nu=1$, respectively. A recent surface transverse acoustic impedance measurement reported in Ref. 48 and 49 revealed the existence of the surface Majorana Dirac fermion mode on the surface of ${ }^{3} \mathrm{He}-\mathrm{B}$.

Many recently found non-centrosymmetric superconductors also fall into class DIII.50 The theory of disordered topological superconductor in class DIII which we will present is also relevant to these materials. A Majorana hopping model on the diamond lattice, which realizes trivial $(\nu=0)$ and non-trivial $(|\nu|=1)$ phases, as well as the Dirac Hamiltonian similar to Eq. (51) was discussed in Ref. 51.

Below, we will consider effects of disorder around this transition separating the weak-strong pairing phases, by perturbing the BdG Hamiltonian (51) by inhomogeneous, time-reversal invariant potential $\mathcal{V}(x)$. With a suitable unitary transformation, the full $\mathrm{BdG}$ Hamiltonian is written in terms of the gamma matrices (2) as

$$
\mathcal{H}=|\Delta|\left(-i \partial_{i}\right) \alpha_{i}+\xi\left(-i \partial_{i}\right) \beta+\mathcal{V}(x)
$$

We will henceforth set $|\Delta|=1$ and drop the $\mathcal{O}\left(k^{2}\right)$ term in $\xi(\mathbf{k}), \xi(\mathbf{k}) \rightarrow-\mu$.

\section{2. class AIII}

Let us consider BdG Hamiltonians which are invariant under rotations about the $z$ - (or any fixed) axis in spin space, $\left[\mathcal{H}_{4}, J_{z}\right]=0$. This implies that the Hamiltonian 
can be brought into the form:

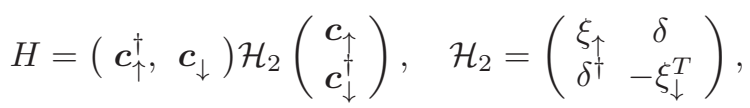

where $\xi_{\sigma}$ and $\delta$ are an $N \times N$ matrix and $\xi_{\sigma}^{\dagger}=\xi_{\sigma}$. Without further constraints, this Hamiltonian is a member of class A (unitary symmetry class). If, in addition to conservation of the $z$-component of spin, we further impose TRS, we obtain the conditions $\xi_{\uparrow}^{T}=\xi_{\downarrow}$ and $\delta=\delta^{\dagger}$, which can be summarized as

$$
r_{y} \mathcal{H}_{2} r_{y}=-\mathcal{H}_{2},
$$

where the Pauli matrices $r_{x, y, z}$ are acting on the particlehole grading. It is also convenient to rewrite the Hamiltonian by rotating the $r_{x, y, z}$ matrices by $\left(r_{x}, r_{y}, r_{z}\right) \Rightarrow$ $\left(r_{x},-r_{z}, r_{y}\right)$. In this basis, the class AIII BdG Hamiltonian takes on block off-diagonal form

$$
\mathcal{H}_{2}=\left(\begin{array}{cc}
0 & D \\
D^{\dagger} & 0
\end{array}\right)
$$

While we are not aware of any (lattice) BdG Hamiltonian realizing topological superconductor in class AIII, an electronic lattice model of class AIII with non-trivial topological charge $\nu=1$ is constructed in Ref. 16. In the continuum, the model can be described in terms of the following massive Dirac Hamiltonian:

$$
\mathcal{H}_{0}=-i \partial_{k} \alpha_{k}-i \beta \gamma^{5} m_{5} .
$$

\section{3. class $C I$}

If, in addition to $\left[\mathcal{H}_{4}, J_{z}\right]=0$, we further impose the full $\mathrm{SU}(2)$ rotation symmetry, $\xi_{\sigma}$ and $\delta$ are constrained by $\xi_{\downarrow}=\xi_{\uparrow}=: \xi, \delta=\delta^{T}$. This defines symmetry class C. Imposing both full $\mathrm{SU}(2)$ rotation and TR symmetries, leads, in addition to $\xi_{\downarrow}=\xi_{\uparrow}$ and $\delta=\delta^{T}$, to $\xi^{*}=\xi$, and $\delta^{*}=\delta$. These conditions can be summarized as

$$
r_{y} \mathcal{H}_{2}^{T} r_{y}=-\mathcal{H}_{2}, \quad \mathcal{H}_{2}^{*}=\mathcal{H}_{2} .
$$

This defines symmetry class CI. Combining these two conditions we can obtain a chiral symmetry, $r_{y} \mathcal{H}_{2} r_{y}=$ $-\mathcal{H}_{2}$. By rotating the $r_{\mu}$ matrices as $\left(r_{x}, r_{y}, r_{z}\right) \Rightarrow$ $\left(r_{x},-r_{z}, r_{y}\right)$, the class CI Hamiltonian takes on block offdiagonal form

$$
\mathcal{H}_{2}=\left(\begin{array}{cc}
0 & D \\
D^{\dagger} & 0
\end{array}\right), \quad D=\delta-i \xi=D^{T} .
$$

In three dimensions, class CI is the only class which admits non-trivial topological state with $\mathrm{SU}(2)$ spin rotation symmetry. A BCS Hamiltonian on the diamond lattice which belongs to symmetry class CI and realizes topological superconductor with $\nu=2$ was constructed in Ref. 15. A model on the cubic lattice was also constructedef, and it was shown that a defect in such topological SC accumulate a spin 1/2 degree of freedom at its core.

\section{B. continuum Dirac model and fermionic replica method}

We now start our discussion on the effects of disorder on topological superconductors. Let us start by considering, as a model of a $3 \mathrm{~d}$ topological superconductor in class AIII, a 3d massive Dirac Hamiltonian

$$
\begin{aligned}
\mathcal{H} & =\mathcal{H}_{0}+\mathcal{V}, \\
\mathcal{H}_{0} & =-i \partial_{k} \alpha_{k}-i \beta \gamma^{5} m_{5}, \\
\mathcal{V} & =\left(\begin{array}{cc}
0 & D_{I}(x) \\
D_{I}^{\dagger}(x) & 0
\end{array}\right),
\end{aligned}
$$

where $D_{I}(x)$ in $\mathcal{V}$ represents a disorder potential. For any value of the mass $m_{5}$ and for any realization of disorder, the Hamiltonian (60) satisfies chiral symmetry

$$
\beta \mathcal{H} \beta=-\mathcal{H} .
$$

As discussed in Ref. 2. $\mathcal{H}_{0}$ realizes, depending on the sign of the mass term $m_{5} \in \mathbb{R}$, a topologically trivial and non-trivial superconductor in class AIII, each of which is characterized by the vanishing and nonvanishing, respectively, of the winding number $\nu$ defined for the projector introduced in Ref. 2. We assume $D_{I}(r)$ is maximally random according to the distribution $\propto \exp \left[-\operatorname{tr}_{2}\left(D_{I} D_{I}^{\dagger}\right) /(2 g)\right]$

To discuss effects of disorder, it is convenient to introduce the generating function, $Z=\int \mathcal{D}\left[\psi_{a}^{\dagger}, \psi_{a}\right] \exp (-$ $\left.\int d^{3} x \mathcal{L}\right)$, for the retarded Green's functions, where $\psi_{a}^{\dagger}, \psi_{a}$ with $a=1, \cdots, N_{r}$ is a replicated fermionic variable, and

$$
\mathcal{L}=\sum_{a=1}^{N_{r}} \psi_{a}^{\dagger}\left(i \mathcal{H}+0^{+}\right) \psi_{a} .
$$

We have introduced $N_{r}$ replicas for the quenched disorder averaging, with $N_{r} \rightarrow 0$ at the end of calculation. The $0^{+}>0$ is introduced for the convergence of the functional integral. The Lagrangian enjoys an $\mathrm{U}\left(N_{r}\right)$ symmetry

$$
\psi^{\dagger} \rightarrow \psi^{\dagger} T^{\dagger}, \quad \psi \rightarrow T \psi, \quad T \in \mathrm{U}\left(N_{r}\right) .
$$

Due to the chiral symmetry of class AIII, there is an additional (axial) $\mathrm{U}\left(N_{r}\right)$ symmetry when $0^{+}=0$,

$$
\psi^{\dagger} \rightarrow \psi^{\dagger} e^{+i \beta \theta}, \quad \psi \rightarrow e^{+i \beta \theta} \psi, \quad \theta \in \mathrm{u}\left(N_{r}\right) .
$$

Averaging over disorder gives

$$
\begin{aligned}
\mathcal{L}= & \sum_{a} \bar{\psi}_{a}\left(\gamma^{k} \partial_{k}-i 0^{+} \gamma^{0}-i m_{5} \gamma^{5}\right) \psi_{a} \\
& -\frac{g}{2} \sum_{a, b}\left(\bar{\psi}_{a} \psi_{b} \bar{\psi}_{b} \psi_{a}-\bar{\psi}_{a} \gamma^{0} \psi_{b} \bar{\psi}_{b} \gamma^{0} \psi_{a}\right),
\end{aligned}
$$

where $\bar{\psi}=\psi^{\dagger} \beta i$ and $\gamma^{k}=-i \beta \alpha^{k}$. [Observe that we introduced $\gamma^{k}$ differently from the case of symmetry class AII, Eq. (25). For symmetry class AIII, DIII, and CI, we 
find this convention is more convenient.] This is a $3 \mathrm{~d}$ version of the chiral Gross-Neveu or the Nambu-JonaLacinio mode 52 perturbed by a mass term.

The quartic interactions among fermions can be decoupled by the Hubbard-Stratonovich transformation with two Hermitian matrices $W$ and $V$,

$$
\begin{aligned}
\mathcal{L}= & \sum_{a} \bar{\psi}_{a}\left(\gamma^{k} \partial_{k}-i 0^{+} \gamma^{0}-i m_{5} \gamma^{5}\right) \psi_{a} \\
& +\sum_{a, b} \bar{\psi}_{a}\left(W_{a b}+i \gamma^{0} V_{a b}\right) \psi_{b} \\
& +\frac{1}{2 g} \operatorname{tr}_{N_{r}}\left(W^{2}+V^{2}\right) .
\end{aligned}
$$

There are similar Dirac type Hamiltonians describing $3 \mathrm{~d}$ topological insulators in class CI and DIII, and the following mean-field and large- $N_{f}$ analysis are completely parallel for these classes. In the large- $N_{f}$ calculations, the nature of Nambu-Goldstone fluctuations are different among symmetry classes AIII, DIII, and CI: Roughly, one needs to replace $\mathrm{U} \rightarrow \mathrm{O}$ for class DIII, and $\mathrm{U} \rightarrow$ Sp for class CI.

\section{C. large $N_{f}$-analysis}

We now look for a spatially homogeneous, replica symmetric saddle point solution, by setting

$$
W_{a b}=w \delta_{a b}, \quad V_{a b}=v \delta_{a b} .
$$

It is enough to look for a saddle point with $v \neq 0$ and $w=$ 0 , since there is a symmetry that relates a saddle point with $w=0$ and $v \neq 0$ and a saddle point with $w \neq 0$ and $v=0$. The resulting mean-field (self-consistent Born) phase diagram is essentially the same as Fig. 3. (We need to replace $\left.m \rightarrow m_{5}\right)$. We identify the phase with $v \neq 0$ (the ordered phase of the $\mathrm{NL} \sigma \mathrm{M}$ ) as a (diffusive) metallic phase. This metallic phase appears because the disorder creates states that fill the band gap. While it is not depicted here, in the large $g$ limit, there should be an Anderson insulating phase in class CI and class DIII. On the other hand, in class AIII, it is not necessary to have an Anderson insulator in the large $g$ limit since the beta function in $(2+\epsilon)$ dimensions is positive to all order in perturbation theory 53 as known in the context of the random hopping (flux) model in one and two dimensions where there is no Anderson localization. For a numerical study of the $3 \mathrm{~d}$ random hopping model in class AIII, See Ref. 54. (This result should be considered, however, as the case with zero topological angle, $\theta=0$, while our system is located at $\theta=\pi / 2$ when $m_{5}=0$; see below for more discussion.)

Just at the Dirac point, the disorder, which appears as a four fermion interaction in the generating function, is irrelevant, whereas the mass term is relevant, from the power-counting. The transition between trivial and topological insulators is then described by the clean Dirac point.
In the large- $N_{f}$ expansion, the model can be renormalized in a similar way as the class AII Gross-Neveu model (25). We introduce wavefunction renormalizations $Z_{\psi}$ and $Z_{W}$ and consider the Lagrangian

$$
\begin{aligned}
\mathcal{L}= & Z_{\psi} \bar{\psi}_{\iota a}\left(\partial_{k} \gamma_{k} \delta_{a b}+\Sigma\right) \psi_{\iota b} \\
& +\frac{g}{\sqrt{N_{f}}} Z_{\psi} Z_{W}^{1 / 2} \bar{\psi}_{\iota a}\left(W_{a b}+i \gamma^{5} V_{a b}\right) \psi_{\iota b} \\
& +\frac{1}{2} Z_{W}\left(W_{a b} W_{a b}+V_{a b} V_{a b}\right),
\end{aligned}
$$

where we have rescaled bosonic fields properly. To leading order, the fermion propagator and the boson propagator are given by

$$
\begin{aligned}
\left\langle\psi_{\iota a}(k) \bar{\psi}_{\kappa b}(k)\right\rangle & =\delta_{\iota \kappa} \delta_{a b} Z_{\psi}^{-1}\left(i k_{i} \gamma_{i}+\Sigma\right), \\
\left\langle W_{a b}(q) W_{c d}\left(-q^{\prime}\right)\right\rangle & =\left\langle V_{a b}(q) V_{c d}\left(-q^{\prime}\right)\right\rangle \\
& =\frac{4}{Z_{W} g^{2} D_{\gamma}} \mathcal{D}\left(q^{2}\right) \delta_{q, q^{\prime}} \delta_{c b} \delta_{d a} .
\end{aligned}
$$

As in the class AII Gross-Neveu model, several critical exponents can be readily computed within the large- $N_{f}$ approach. For example, the anomalous dimensions of a fermion $\psi$ to leading order in $1 / N_{f}$ is given by

$$
\eta_{\psi}=\alpha=\frac{16 N_{r}}{3 \pi^{2} N_{f} D_{\gamma}} \quad \text { for class AIII, }
$$

where the fermion Green's function behaves as $\mathcal{G}(x) \sim$ $|x|^{-2-\eta_{\psi}}$. In the replica limit, this leading order correction is vanishing. Similarly, the anomalous dimension of the fermion mass is given by

$$
\eta_{\bar{\psi} \psi}=\frac{32 N_{r}}{3 \pi^{2} N_{f} D_{\gamma}}
$$

and the anomalous dimension of the " $\gamma_{5}$ " fermion mass is given by

$$
\eta_{\bar{\psi} \gamma_{5} \psi}=\frac{32 N_{r}}{\pi^{2} N_{f} D_{\gamma}} .
$$

The similar calculations go through for class DIII, and CI. For symmetry class DIII,

$$
\eta_{\psi}=\frac{64 N_{r}}{3 \pi^{2} N_{f} D_{\gamma}}
$$

and for the anomalous dimension of the fermion mass is given by

$$
\eta_{\bar{\psi} \psi}=\frac{256\left(N_{r}-3\right)}{3 \pi^{2} N_{f} D_{\gamma}}
$$

\section{D. non-linear sigma models}

Deep in the diffusive metallic phase, physics at large distances should be described by the NL $\sigma \mathrm{M}$ defined on 


\begin{tabular}{ccc}
\hline \hline $\begin{array}{c}\text { symmetry } \\
\text { class }\end{array}$ & $\begin{array}{c}\mathrm{NL} \sigma \mathrm{M} \\
\text { target space }\end{array}$ & $\begin{array}{c}\text { 3d topological } \\
\text { term }\end{array}$ \\
\hline A & $\mathrm{U}(2 N) / \mathrm{U}(N) \times \mathrm{U}(N)$ & \\
AI & $\mathrm{Sp}(2 N) / \mathrm{Sp}(N) \times \mathrm{Sp}(N)$ & \\
AII & $\mathrm{O}(2 N) / \mathrm{O}(N) \times \mathrm{O}(N)$ & \\
\hline AIII & $\mathrm{U}(N) \times \mathrm{U}(N) / \mathrm{U}(N)$ & \\
BDI & $\mathrm{U}(2 N) / \mathrm{Sp}(N)$ & $\mathbb{Z}_{2}$ \\
CII & $\mathrm{U}(2 N) / \mathrm{O}(2 N)$ & \\
$\mathrm{D}$ & $\mathrm{O}(2 N) / \mathrm{U}(N)$ & $\mathbb{Z}_{2}$ \\
$\mathrm{C}$ & $\mathrm{Sp}(N) / \mathrm{U}(N)$ & $\mathbb{Z}$ \\
$\mathrm{DIII}$ & $\mathrm{O}(N) \times \mathrm{O}(N) / \mathrm{O}(N)$ & $\mathbb{Z}$ \\
CI & $\mathrm{Sp}(N) \times \mathrm{Sp}(N) / \mathrm{Sp}(N)$ & \\
\hline \hline
\end{tabular}

TABLE I. Fermionic replica NL $\sigma$ M target spaces and possible topological terms in three dimensions. For a similar table for two spatial dimensions, see Ref. 55.

$\mathrm{U}\left(N_{r}\right)$ (AIII), $\mathrm{O}\left(2 N_{r}\right)$ (DIII), and $\mathrm{Sp}\left(N_{r}\right)(\mathrm{CI})$, respectively. The coupling constant of the $\mathrm{NL} \sigma \mathrm{M}$ plays the role of the longitudinal conductivity $\sigma_{x x}$. (To be more precise, in superconductors, $\sigma_{x x}$ corresponds to either spin or thermal conductivity.) In addition, one should note here that the NL $\sigma \mathrm{M}$ for these classes AIII, DIII, and CI can support a topological term in three dimensions: For the NL $\sigma \mathrm{M}$ target manifolds $G$, where $G=\mathrm{U}\left(N_{r}\right)$ (AIII), $G=\mathrm{O}\left(2 N_{r}\right)(\mathrm{DIII})$, and $G=\operatorname{Sp}\left(N_{r}\right)(\mathrm{CI})$, the homotopy group is given by $\pi_{3}(G)=\mathbb{Z}$.

The NL $\sigma \mathrm{M}$ and the topological term can be obtained in the following way (we will focus on symmetry class AIII as an example, but a similar derivation should be possible for class DIII and CI): a saddle point solution in Eq. (67) breaks the global $\mathrm{U}\left(N_{r}\right) \times \mathrm{U}\left(N_{r}\right)$ symmetry down to $\mathrm{U}\left(N_{r}\right)$. One then expects the long wave length physics are described in terms of the Nambu-Goldstone modes associated with the symmetry breaking. With the inclusion of the Nambu-Goldstone modes (=fluctuations around the saddle point), the action is

$$
\mathcal{L}=\bar{\psi}_{a}\left[\left(\gamma^{k} \partial_{k}+m_{5} \gamma^{5}\right) \delta_{a b}+q \gamma^{0}\left(e^{2 i \gamma^{0} \theta(x)}\right)_{a b}\right] \psi_{b},
$$

where the slow variations represented by $\exp \left(2 i \gamma^{0} \theta(x)\right)$ are the Nambu-Goldstone modes in the NL $\sigma \mathrm{M}$,

$$
e^{2 i \gamma^{0} \theta(x)}=\left(\begin{array}{cc}
U & 0 \\
0 & U^{\dagger}
\end{array}\right), \quad U \in \mathrm{U}\left(N_{r}\right) .
$$

The Lagrangian (75) is obtained from Eq. (66) by freezing the longitudinal fluctuations but keeping the transverse fluctuations (i.e., the Nambu-Goldstone modes). The longitudinal fluctuations are gapped and can safely be integrated over, which can renormalize parameters in the effective action. (In symmetry class AIII, it can also generate the so-called Gade term.53 We do not discuss, however, the effect of the Gade term in this section.) The effective action $S_{\text {eff }}[U]$ for the Nambu-Goldstone modes is then obtained by integrating over fermions,

$$
Z=\int \mathcal{D}[U] \mathcal{D}[\bar{\psi}, \psi] e^{-S}=\int \mathcal{D}[U] e^{-S_{\text {eff }}[U]}
$$

Expanding the effective action in powers of $U$ and its gradient, and with the Pauli-Villars regularization 56 the effective action (the NL $\sigma \mathrm{M}$ action) is given by

$$
S_{\mathrm{eff}}[U]=\frac{\pi \sigma_{x x}}{4} \int d^{3} x \operatorname{tr}\left(\partial_{j} U^{\dagger} \partial_{j} U\right)+i \theta \Gamma[U],
$$

where the topological term

$$
\Gamma[U]=\int \frac{d^{3} x}{24 \pi^{2}} \epsilon^{i j k} \operatorname{tr}\left(U^{\dagger} \partial_{i} U U^{\dagger} \partial_{j} U U^{\dagger} \partial_{k} U\right)
$$

is an integer for any field configuration. The $\theta$ angle (topological angle) is given by (see Appendix $\mathbf{Q}$ )

$$
\begin{aligned}
\frac{\theta}{\pi} & =-\frac{m_{5}^{3}}{|M|^{3}}+\frac{m_{R}^{3}}{\left|m_{R}\right|^{3}}-\frac{9 m_{5} m_{0}^{2}}{4|M|^{3}}, \\
M^{2} & :=m_{5}^{2}+m_{0}^{2},
\end{aligned}
$$

where $m_{0}$ is the imaginary part of the self-energy (within the self-consistent Born approximation), and $m_{R}$ is a mass introduced by the Pauli-Villars regularization. Notice that the topological angle is $\pi$ when $m_{5}$ is 0 (Dirac point) for arbitrary $m_{0}$.

\section{1. possible two-parameter scaling phase diagram}

The presence of the topological term is a direct manifestation of the fact that the metallic phase is adjacent to a topological superconducting phase. The similar topological term in two dimensions, the Pruisken term in the QHE, has a significant implication on the nature of Anderson localization physics, and, in fact, is crucial for the existence of the QHE, and for the plateau transition. The role of such Pruisken term in topological superconductors with broken time-reversal symmetry in two dimensipen has been discussed in symmetry class A, D, and C. 5759

It is not clear what the role is played by the topological term in $3 \mathrm{~d} \mathrm{NL} \sigma \mathrm{Ms}$. While controlled calculations near metal-insulator transitions and in the presence of topological terms are in general difficult, below we combine the RG flow of the NL $\sigma \mathrm{M}$ for large $\sigma_{x x}$ with the RG flow around the clean Dirac point from the $1 / N_{f^{-}}$ expansion to speculate the general structure of the phase diagram 60 As in the QHE, the phase diagram and the RG flow may be phrased in terms of the two parameters of the $3 \mathrm{~d} \mathrm{NL} \sigma \mathrm{M}$; the $\mathrm{NI}_{\mathrm{G}} \mathrm{M}$ coupling constant $1 / \sigma_{x x}$ and the topological angle $\theta$. 1 .

We first discuss the phase diagram for class CI and DIII. (Symmetry class AIII has a complication due to the special nature of its beta function in $(2+\epsilon)$ dimensions - see below). The beta functions for these NL $\sigma \mathrm{Ms}$ in $3 \mathrm{~d}$ when $\theta=0$ are expected to have a zero at finite $\sigma_{x x}$, separating stable metallic and Anderson insulating phases. For large $\sigma_{x x}$, the RG flow for arbitrary $\theta \neq 0$ is expected to be similar to the RG flow at $\theta=0$, while the flow for $\theta \neq 0$ and for small $\sigma_{x x}$ needs to be determined. In terms of the massive Dirac model with disorder, on the 


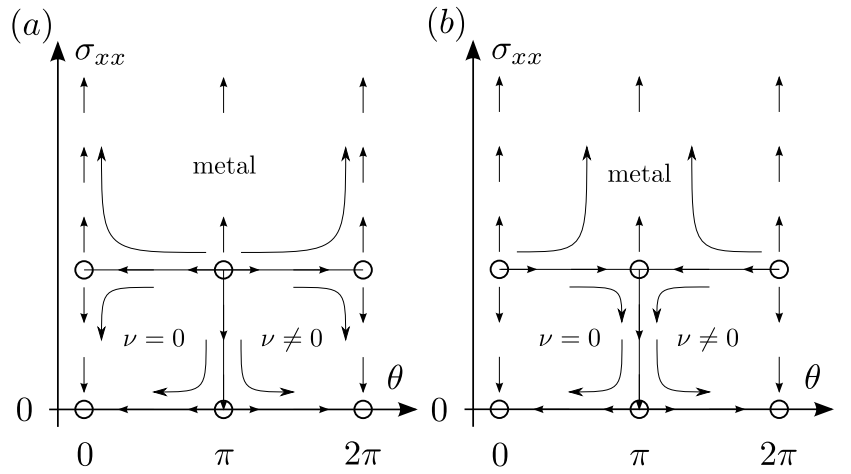

FIG. 5. Possible two-parameter scaling flow diagrams of symmetry class CI and DIII.

other hand, $\sigma_{x x}$ is small around the clean Dirac fermion point (the dc conductivity of the clean $3 \mathrm{~d}$ Dirac fermion is zero) and $\theta \sim \pi$. It is thus tempting to "identify" the point $\left(\sigma_{x x}, \theta\right)=(0, \pi)$ in the NL $\sigma \mathrm{M}$ phase diagram as the clean Dirac fixed point, or, at least, to use the Dirac model (i.e., the chiral Gross-Neveu model) to guess the RG flow of the NL $\sigma \mathrm{M}$.

The fermion mass term $\bar{\psi} \gamma_{5} \psi$ in the chiral Gross-Neveu model is a relevant perturbation to the clean Dirac fixed point. Also, depending on the sign of the mass, one can drive the clean Dirac fermion either into the topological $(\nu \neq 0)$ or trivial $(\nu=0)$ insulating phase. As the fermion mass corresponds to the theta term in the $\mathrm{NL} \sigma \mathrm{M}$, this would suggest the deviation of the theta angle from $\theta=\pi$ is relevant at $\sigma_{x x}=0$. On the other hand, disorder at the clean Dirac fixed point is irrelevant by power-counting. Furthermore, the existence of the UV fixed point (the Gross-Neveu fixed point) in the $1 / N_{f}$-expansion of the chiral Gross-Neveu model would suggest the existence of a corresponding critical point at $\theta=\pi$ in the $\mathrm{NL} \sigma \mathrm{M}$ (Fig. [) separating a metallic phase and an insulating phase (which is adiabatically connected the clean 3d Dirac fermion with $\sigma_{x x}=0$ ).

While we expect the deviation of the theta angle from $\theta=\pi$ is relevant at $\sigma_{x x}=0$ (i.e., at the clean Dirac fixed point), there is an ambiguity on the RG flow around the putative critical point at $\theta=\pi$ and $\sigma_{x x} \neq 0$. If it is relevant, as it is at the clean Dirac point $\left(\sigma_{x x}, \theta\right)=(0, \pi)$, the flow looks like Fig. F-(a). An alternative scenario where the theta term is irrelevant at the Gross-Neveu critical point is depicted in Fig. 5 (b). In these scenarios, an implicit assumption we have made is that there are no other fixed points. Also, we have not considered more exotic possibilities, such as the case where $\theta$ is exactly marginal at the Gross-Neveu fixed point. Which one of the two scenarios is realized may be speculated, again, from the large $N_{f}$ approach, by computing the anomalous dimension of the $\gamma_{5}$ fermion mass; See Eqs. (71), 72), and (73) for relevant calculation. We should, of course, bear in mind that the structure of the phase diagram would be quite different for large $N_{f}$ and for small $N_{f}$ (e.g., $N_{f} \rightarrow 1$ ). In other words, with the inclusion of the $N_{f}$ flavor of Dirac fermions, the model describes, in the absence of disorder, a transition between trivial and topological insulators with $\nu=N_{f}$. This is a highly fine tuned critical point, and might be quite different from the critical point that may exist and be described in the NL $\sigma \mathrm{M}$.

Symmetry class AIII is special in that the beta function in $(2+\epsilon)$-expansion is always positive; Thus, at $\theta=0$, there is no metal insulator transition, but we always have a metal. However, as the large- $N_{f}$ expansion (which corresponds to $\theta \neq 0$ ) predicts tuning $\theta \neq 0$ seems to generate a metal-insulator transition. Because of the positivity of the beta function in $(2+\epsilon)$ dimensions, it appears that constructing a reasonable conjecture on the two-parameter scaling phase diagram is more complicated.

\section{DISCUSSION}

Topological insulators and superconductors are known to be an inherently holographic system; The non-trivial bulk topology [as encoded by a non-vanishing bulk topological invariant such as the Chern (TKNN) 6 integer or the $\mathbb{Z}_{2}$ invariant in $\mathbb{Z}_{2}$ topological insulators] has a crucial impact on the Anderson localization problem at boundaries of the system; in fact, Anderson localization is not allowed at the system's boundaries because of topological reasons.63 65 Such bulk-boundary correspondence served as a guiding principle of classification of topological insulators and superconductors. In this paper, we have investigated Anderson localization problem in the bulk of topological insulators and superconductors in three dimensions; we have explored implications of bulk topology on physics of Anderson localization in the bulk.

In the QHE, the transition between topological phases with different Chern integers is governed by a disorderdominated quantum critical point (the plateau transition). The fact that delocalized (extended) states are possible at the plateau transition is a direct manifestation of a topological character of the system, which is not allowed otherwise in two dimensions with broken timereversal symmetry. In the NL $\sigma \mathrm{M}$ description of the QHE, the plateau transition is encoded in the Pruisken term (which also points the existence of edge modes). We can draw a rather close analogy between time-reversal symmetric topological superconductors in three dimensions and the 2d QHE, both of which are characterized by an integer topological invariant in the bulk. We have given a field theory description of metal-insulator transition in terms of the Dirac model of topological superconductors by employing the large- $N_{f}$ expansion, and also in terms of the NL $\sigma \mathrm{Ms}$. Although the presence of topological terms in the NL $\sigma \mathrm{Ms}$ is indicative, it is an open question if the phase diagram is characterized in terms of two parameter scaling, as in the QHE. It is desirable to explore further the structure of the phase diagram and critical behaviors in $3 \mathrm{~d}$ topological superconductor sys- 
tems in terms of, say, numerics, preferably in the presence of the non-zero theta angle in the language of the NL $\sigma \mathrm{M}$. This would require more microscopic and physical understanding of the theta angle; e.g., how one should interpret and measure the theta term in a metallic phase.66.67

Our discussion for symmetry class DIII can be relevant, for example, for ${ }^{3} \mathrm{He}$ in aerogel, 68.69 although we need to include pair breaking effects. Antalso $\mathrm{Cu}$-intercalated topological insulators, $\mathrm{Cu}_{x} \mathrm{Bi}_{2} \mathrm{Se}_{3}, 7073$ and many heavy fermion non-centrosymmetric superconductors with antisymmetric spin-orbit coupling 50 Experimental probes for detecting these systems are, for example, thermal transport, and spin transport spin or part of spin quantum number is conserved.745 See, for example, for the anomalous thermal Hall effect by magnons, Ref. 76.

In the $3 \mathrm{~d} \mathbb{Z}_{2}$ topological insulator in symplectic symmetry class, a closer analogy would be the $2 \mathrm{~d}$ quantum spin Hall effect. We have numerically investigated the structure of the phase diagram by the transfer matrix method, and developed the large- $N_{f}$ field theory approach to a metal-insulator transition. It should be noted, however, the large $N_{f}$ expansion does not distinguish the case of odd $N_{f}$, for which the one of the insulating states in the phase diagram Fig. 3 is a topologically non-trivial, $\mathbb{Z}_{2}$ topological insulator, and the case of even $N_{f}$, for which the one of the insulating states in the phase diagram Fig. 3 is a topologically trivial. While we have imposed the global $\mathrm{O}\left(N_{f}\right)$ symmetry in the large $N_{f}$ expansion, perhaps a distinction between even and odd $N_{f}$ would emerge once we study the stability of the UV fixed point (the Gross-Neveu fixed point) that can be reached by the large $N_{f}$ expansion; for example, for even $N_{f}$, the Gross-Neveu fixed point is unstable against $\mathrm{O}\left(N_{f}\right)$ symmetry breaking (and other) perturbations, and the metal-insulator transition is always controlled by the conventional critical point of symplectic symmetry class. In terms of the NL $\sigma \mathrm{M}$ description, there is no topological term of any kind in the bulk in symmetry class AII, and hence the transition between the metal and insulating phases is expected in the conventional universality class of symplectic symmetry class, irrespective of the number of flavors. This situation is similar to the $\mathbb{Z}^{\text {topological }}$ insulators in class AII in two dimensions29. 30.

While we have discussed the bulk critical behavior, it would be interesting to study boundary critical behaviors; even when a transition between a trivial insulator to a metal and one between a topological insulator to a metal is in the same universality class, at a surface we might observe different critical behavior. This was indeed the case in the $2 \mathrm{~d}$ symplectic class. 77

Finally, we conclude with some remarks on other symmetry classes in three dimensions.

- For symmetry class CIf a $\mathbb{Z}_{2}$ topological insulator can be realized in the bulk.16 While we did not discuss symmetry class CII, a similar field theory analysis can be developed.

- The large- $N_{f}$ technique developed here is also applicable to the Weyl semimetal in symmetry class A, which are fealized in the A phase of ${ }^{3} \mathrm{He}$ and Pyrochlore Iridates.78.79

- We mention two other symmetry classes for which the corresponding $\mathrm{NL} \sigma \mathrm{M}$ has a topological term of $\mathbb{Z}_{2}$ type: in symmetry class $\mathrm{C}$ and $\mathrm{CII}, \mathbb{Z}_{2}$ insulators (superconductors) can be realized in four dimensions. Thus, the $3 \mathrm{~d} \mathbb{Z}_{2}$ topological term in the corresponding NL $\sigma \mathrm{M}$ describes the surface of $4 \mathrm{~d}$ insulator, but not any isolated 3 d system.

\section{ACKNOWLEDGMENTS}

The authors acknowledge helpful interactions with Hideaki Obuse, and Ai Yamakage. S.R. thanks RIKEN for hospitality. This research is granted by the Japan Society for the Promotion of Science (JSPS) through the "Funding Program for World-Leading Innovative R\&D on Science and Technology (FIRST Program)," initiated by the Council for Science and Technology Policy (CSTP).

\section{Appendix A: transfer matrix}

a. transfer matrix In this appendix, we derive the transfer matrix for four-component 3d Dirac fermions. The Dirac Hamiltonian is written in the single-particle representation as

$$
\begin{aligned}
H= & -\sum_{n, \mathbf{r}}\left[|\mathbf{r}, n+1, \sigma\rangle u_{-, \sigma \sigma^{\prime}}^{z}\left\langle\mathbf{r}, n, \sigma^{\prime}\right|\right. \\
& \left.+|\mathbf{r}, n, \sigma\rangle u_{+, \sigma \sigma^{\prime}}^{z}\langle\mathbf{r}, n+1, \sigma|\right] \\
- & \sum_{n, \mathbf{r}} \sum_{\mu=1,2}\left[\left|\mathbf{r}+\mathbf{e}_{\mu}, n, \sigma\right\rangle u_{-, \sigma \sigma^{\prime}}^{\mu}\left\langle\mathbf{r}, n, \sigma^{\prime}\right|\right. \\
& \left.+|\mathbf{r}, n, \sigma\rangle u_{+, \sigma \sigma^{\prime}}^{\mu}\left\langle\mathbf{r}+\mathbf{e}_{\mu}, n, \sigma^{\prime}\right|\right] \\
+ & \sum_{n, \mathbf{r}}|\mathbf{r}, n, \sigma\rangle\left[(m+3) \beta+\varepsilon_{\mathbf{r} n}\right]_{\sigma \sigma^{\prime}}\left\langle\mathbf{r}, n, \sigma^{\prime}\right|,
\end{aligned}
$$

where we have introduced

$$
u_{ \pm}^{\mu}=\frac{1}{2}\left(\beta \pm i t \alpha_{\mu}\right) .
$$

The eigen wave function with energy $E$ of this Hamiltonian satisfies

$$
\begin{aligned}
0= & \langle\mathbf{r} n \sigma|(H-E)| \psi\rangle \\
= & -u_{-, \sigma \sigma^{\prime}}^{z} \psi_{\sigma^{\prime}}(\mathbf{r}, n-1)-u_{+, \sigma \sigma^{\prime}}^{z} \psi_{\sigma^{\prime}}(\mathbf{r}, n+1) \\
& +\left[\mathcal{H}^{(n)}-E\right]_{\sigma \sigma^{\prime}} \psi_{\sigma^{\prime}}(\mathbf{r}, n)
\end{aligned}
$$

which leads to

$$
\begin{aligned}
\psi_{n+1}= & 2\left(\beta+i t \alpha_{z}\right)^{-1}\left[\mathcal{H}^{(n)}-E\right] \psi_{n} \\
& -\left(\beta+i \alpha_{z}\right)^{-1}\left(\beta-i \alpha_{z}\right) \psi_{n-1}
\end{aligned}
$$


Note that $\left(\beta+i t \alpha_{z}\right)^{-1}=\left(\beta+i t \alpha_{z}\right) /\left(1-t^{2}\right)$, and Eq. (7) is obtained. The transfer matrix $M^{(n)}$ is defined as

$$
\left(\begin{array}{c}
\psi_{n+1} \\
\psi_{n}
\end{array}\right)=M^{(n)}\left(\begin{array}{c}
\psi_{n} \\
\psi_{n-1}
\end{array}\right), \quad M^{(n)}=\left(\begin{array}{cc}
h^{(n)} & v \\
1 & 0
\end{array}\right),
$$

where

$$
\begin{aligned}
h^{(n)} \equiv & \frac{-4}{t^{2}-1} u_{+}^{z}\left[\mathcal{H}^{(n)}-E\right] \\
= & \sum_{n, \mathbf{r}, \mu=1,2}\left|\mathbf{r}+\mathbf{e}_{\mu}, n, \sigma\right\rangle\left[\frac{4 u_{+}^{z} u_{-}^{z}}{t^{2}-1}\right]_{\sigma \sigma^{\prime}}\left\langle\mathbf{r}, n, \sigma^{\prime}\right| \\
& +\sum_{n, \mathbf{r}, \mu=1,2}|\mathbf{r}, n, \sigma\rangle\left[\frac{4 u_{+}^{z} u_{+}^{\mu}}{t^{2}-1}\right]_{\sigma \sigma^{\prime}}\left\langle\mathbf{r}+\mathbf{e}_{\mu}, n, \sigma^{\prime}\right| \\
& +\sum_{n, \mathbf{r}}|\mathbf{r}, n, \sigma\rangle \frac{-4}{t^{2}-1}\left[(m+3) u_{+}^{z} \beta\right. \\
& \left.\left.+\left(\varepsilon_{\mathbf{r} n}-E\right) u_{+}^{z}\right]_{\sigma \sigma^{\prime}}\left\langle\mathbf{r}, n, \sigma^{\prime}\right| . \quad \text { (A }\right)
\end{aligned}
$$

Appendix B: large- $N_{f}$ expansion for three-dimensional topological superconductors

In this Appendix, we illustrate the large- $N_{f}$ calculations for $3 \mathrm{~d}$ time-reversal symmetric topological superconductors in symmetry class AIII, DIII, and CI. We start, in Subsec. B 1, by reviewing fermionic replica methods for symmetry class AIII, DIII, and CI. The formalism in Subsec. B1, is general and applies to any systems in these symmetry classes in any dimensions other than $3 \mathrm{~d}$ Dirac representatives of topological superconductors discussed in the main text. In Subsec. B 2, we introduce auxiliary matrix fields (Hubbard-Stratonovich fields) to decouple four fermion interactions induced by quenched disorder averaging, which is useful to develop the large$N_{f}$ expansion, and also to derive the NL $\sigma \mathrm{M}$ inside metallic phases.

\section{1. fermionic replica field theories}

For symmetry classes AIII, DIII, and CI, the Hamiltonians anticommute with a unitary matrix $\Gamma_{z}$ with equal number of eigenvalues +1 and $-1,\left\{\mathcal{H}, \Gamma_{z}\right\}=0$, thereby they can be brought into an block off-diagonal form,

$$
\mathcal{H}=\left(\begin{array}{cc}
0 & D \\
D^{\dagger} & 0
\end{array}\right)
$$

in the basis where $\Gamma_{z}$ is written diagonally as

$$
\Gamma_{z}=\left(\begin{array}{cc}
1 & 0 \\
0 & -1
\end{array}\right) \text {. }
$$

For class AIII, the Hamiltonians are not subjected to any further constraint. On the other hand, for classes DIII and CI, the off-diagonal block $D$ satisfies

$$
\begin{array}{ll}
D^{T}=-D & \text { for class DIII, } \\
D^{T}=+D & \text { for class CI }
\end{array}
$$

In the following, we will assume that the Hamiltonian consists of the disorder-free $\left(\mathcal{H}_{0}\right)$ and disordered $(\mathcal{V})$ parts,

$$
\mathcal{H}=\mathcal{H}_{0}+\mathcal{V}, \quad D=D_{0}+D_{I}(x),
$$

where the random potential $\mathcal{V}$ is assumed to be whitenoise and to be maximally random according to the distribution

$$
\propto \exp \left[-\operatorname{tr}\left(D_{I} D_{I}^{\dagger}\right) /(2 g)\right]
$$

with $g$ characterizing the strength of disorder.

In order to study Anderson localization physics, we are after the properties of the single particle Green's function $G(z)=(z-\mathcal{H})^{-1}$ at zero energy, Re $z=0$. Since "chiral" symmetry $\left\{\mathcal{H}, \Gamma_{z}\right\}=0$ relates retarded and advanced Green's functions at zero energy, we can focus either one of them, the retarded Green's function, say, $G\left(z=i 0^{+}\right)=\left(i 0^{+}-\mathcal{H}\right)^{-1}$, where $0^{+}>0$ is positive infinitesimal. The generating function for the retarded Green function can be expressed in terms of fermionic functional integral, $Z=\int \mathcal{D}\left[f^{\dagger}, f\right] \exp \left(-\int d^{3} x \mathcal{L}\right)$,

$$
\mathcal{L}=f^{\dagger}\left(i \mathcal{H}+0^{+}\right) f
$$

where $f^{\dagger}$ and $f$ are independent fermionic path integral variables.

To perform the $1 / N_{f}$ expansion, we generalize the above functional integral with single flavor to $Z=$ $\int \mathcal{D}\left[f_{\iota}^{\dagger}, f_{\iota}\right] \exp \left(-\int d^{3} x \mathcal{L}\right)$

$$
\mathcal{L}=\sum_{\iota, \kappa=1}^{N_{f}} f_{\iota}^{\dagger}\left[\left(i \mathcal{H}_{0}+0^{+}\right) \delta_{\iota \kappa}+i \mathcal{V}_{\iota \kappa}\right] f_{\kappa}
$$

where the disorder part

$$
\mathcal{V}_{\iota \kappa}=\left(\begin{array}{cc}
0 & D_{I, \iota \kappa} \\
D_{I, \iota \kappa}^{\dagger} & 0
\end{array}\right)
$$

is a suitable generalization of $\mathcal{H}_{I}$ which couples all flavors equally according to the disorder distribution $\propto \exp [-$ $\left.\operatorname{tr}\left(D_{I, \iota \kappa} D_{I, \kappa \iota}^{\dagger}\right) /(2 g)\right]$.

\section{a. class AIII}

Quenched averaging over disorder $D_{I}$ can be done by introducing replicas $\left\{f_{a \iota}^{\dagger}, f_{a \iota}^{\dagger}\right\}_{a=1, \ldots, N_{r}}$ which leads to the replicated Lagrangian

$$
\begin{aligned}
\mathcal{L}_{\mathrm{AIII}} & =\psi_{a \iota}^{\dagger}\left(i \mathcal{H}_{0}+0^{+}\right) \psi_{a \iota} \\
& -\frac{g}{2}\left(\psi_{a \iota}^{\dagger} \psi_{b \iota} \psi_{b \kappa}^{\dagger} \psi_{a \kappa}-\psi_{a \iota}^{\dagger} \Gamma_{z} \psi_{b \iota} \psi_{b \kappa}^{\dagger} \Gamma_{z} \psi_{a \kappa}\right),
\end{aligned}
$$

where we have renamed $f^{\dagger}, f \rightarrow \psi^{\dagger}, \psi$, and repeated replica and flavor indices are implicitly summed. 
When we deal with the relativistic model (massive Dirac model) of the topological superconductor, it will prove convenient to introduce

$$
\bar{\psi}:=\psi^{\dagger} i \Gamma_{z} .
$$

The Lagrangian is then given as

$$
\begin{aligned}
& \mathcal{L}_{\mathrm{AIII}}=\bar{\psi}_{a \iota}\left(\Gamma_{z} \mathcal{H}_{0}-i \Gamma_{z} 0^{+}\right) \psi_{a \iota} \\
& \quad+\frac{g}{2}\left(\bar{\psi}_{a \iota} \Gamma_{z} \psi_{b \iota} \bar{\psi}_{b \kappa} \Gamma_{z} \psi_{a \kappa}-\bar{\psi}_{a \iota} \psi_{b \iota} \bar{\psi}_{b \kappa} \psi_{a \kappa}\right)
\end{aligned}
$$

This is the chiral Gross-Neveu model with $\mathrm{U}\left(N_{r}\right) \times \mathrm{U}\left(N_{r}\right)$ internal symmetry.

\section{b. class DIII}

For class DIII and (and class CI, which will be discussed later), it is advantageous to double the number of components of fermionic fields to account for the symmetry of the Hamiltonian, $D= \pm D^{T}$. For class DIII, we first note that

$$
\begin{aligned}
& \int d^{3} x f_{A}^{\dagger} D f_{B}=+\int d^{3} x f_{B}^{T} D f_{A}^{*}, \\
& \int d^{3} x f_{B}^{\dagger} D^{\dagger} f_{A}=+\int d^{3} x f_{A}^{T} D^{\dagger} f_{B}^{*},
\end{aligned}
$$

where $f=\left(f_{A}, f_{B}\right)^{T}$ in the chiral (or "sublattice") grading. Introducing the "charge-conjugation" space by

$$
\chi_{+}=\frac{1}{\sqrt{2}}\left(\begin{array}{c}
f_{B} \\
f_{A}^{*}
\end{array}\right), \quad \chi_{-}=\frac{1}{\sqrt{2}}\left(\begin{array}{c}
f_{A} \\
f_{B}^{*}
\end{array}\right),
$$

the Lagrangian can be written as

$$
\begin{aligned}
\mathcal{L}_{\text {DIII }}= & \chi_{+}^{T} i \tau_{x} \otimes D \chi_{+}+\chi_{-}^{T} i \tau_{x} \otimes D^{\dagger} \chi_{-} \\
& +\frac{0^{+}}{2}\left(\chi_{+}^{T} \tau_{x} \tau_{z} \chi_{-}+\chi_{-}^{T} \tau_{x} \tau_{z} \chi_{+}\right)
\end{aligned}
$$

where the Pauli matrices $\tau_{0, x, y, z}$ acts on the "chargeconjugation" space. Further redefining the fermionic fields as

$$
\begin{array}{ll} 
& \eta_{+}:=\tau_{z y} \chi_{+}, \quad \eta_{-}:=\tau_{z y} \chi_{-}, \\
\text {where } \quad \tau_{z y}=\left(\tau_{z}+\tau_{y}\right) / \sqrt{2}, \quad \tau_{z y}^{T} \tau_{z y}=-i \tau_{x},
\end{array}
$$

the Lagrangian can be written as

$$
\begin{aligned}
\mathcal{L}_{\mathrm{DIII}}= & i\left(i \eta_{+}^{T} \tau_{0} \otimes D \eta_{+}+i \eta_{-}^{T} \tau_{0} \otimes D^{\dagger} \eta_{-}\right) \\
& +\frac{0^{+}}{2}\left(i \eta_{+}^{T} \tau_{y} \eta_{-}+i \eta_{-}^{T} \tau_{y} \eta_{+}\right),
\end{aligned}
$$

where we noted $\tau_{z y} \tau_{z} \tau_{z y}=\tau_{y}$.

At this stage, we notice that $f^{\dagger} \mathcal{H} f$ is invariant under $\mathrm{O}(2) \times \mathrm{O}(2)$ transformations,

$$
\eta_{+} \rightarrow O_{+} \eta_{+}, \quad \eta_{-} \rightarrow O_{-} \eta_{-},
$$

where $O_{ \pm} \in \mathrm{O}(2)$. On the other hand, the "smearing term" $f^{\dagger} 0^{+} f$ breaks it down to $\mathrm{O}(2)$. Indeed, in order for $O_{+}$and $O_{-}$to leave the smearing term invariant, they cannot be independent and must be "locked" by $O_{-}=-i \tau_{y} O_{+} i \tau_{y}$, where $i \tau_{y} \in \mathrm{O}(2)$. With replicas, the $\mathrm{O}(2) \times \mathrm{O}(2)$ symmetry is promoted to $\mathrm{O}\left(2 N_{r}\right) \times \mathrm{O}\left(2 N_{r}\right)$ symmetry which is broken down to $\mathrm{O}\left(2 N_{r}\right)$ by the smearing (see below).

Introducing the notation,

$$
\psi^{\dagger}=i\left(\eta_{+}^{T}, \eta_{-}^{T}\right), \quad \psi=\left(\begin{array}{c}
\eta_{-} \\
\eta_{+}
\end{array}\right),
$$

the Lagrangian is written as

$$
\mathcal{L}_{\text {DIII }}=\psi^{\dagger}\left(i \tau_{0} \otimes \mathcal{H}_{0}+0^{+} \tau_{y} \otimes I\right) \psi,
$$

where we have rescaled $0^{+} / 2 \rightarrow 0^{+}$. Observe that $\psi^{\dagger}$ and $\psi$ satisfy the "Majorana" condition

$$
\psi^{T} i \Gamma_{x}=\psi^{\dagger} .
$$

With $N_{f}$ flavors and $N_{r}$ replicas, the Lagrangian after quenched disorder averaging can be cast into the form similar to the one for class AIII,

$$
\begin{aligned}
\mathcal{L}_{\mathrm{DIII}}= & \psi_{a \iota}^{\dagger}\left[i \mathcal{H}_{0} \delta_{a b}+0^{+}\left(\tau_{y} \otimes I_{N_{r}}\right)_{a b}\right] \psi_{b \iota} \\
& -\frac{g}{2}\left(\psi_{a \iota}^{\dagger} \psi_{b \iota} \psi_{b \kappa}^{\dagger} \psi_{a \kappa}-\psi_{a \iota}^{\dagger} \Gamma_{z} \psi_{b \iota} \psi_{b \kappa}^{\dagger} \Gamma_{z} \psi_{a \kappa}\right),
\end{aligned}
$$

where $a, b$ represents the combined charge conjugation and replica index running from 1 to $2 N_{r}$.

If we introduce

$$
\bar{\psi}:=\psi^{\dagger} i \Gamma_{z}, \quad \psi^{T} i \Gamma_{y}=\bar{\psi},
$$

the Lagrangian is then given as

$$
\begin{aligned}
& \mathcal{L}_{\mathrm{DIII}}=\bar{\psi}_{a \iota}\left[\Gamma_{z} \mathcal{H}_{0} \delta_{a b}+0^{+} \Gamma_{z}\left(-i \tau_{y}\right)_{a b}\right] \psi_{b \iota} \\
& \quad+\frac{g}{2}\left(\bar{\psi}_{a \iota} \Gamma_{z} \psi_{b \iota} \bar{\psi}_{b \kappa} \Gamma_{z} \psi_{a \kappa}-\bar{\psi}_{a \iota} \psi_{b \iota} \bar{\psi}_{b \kappa} \psi_{a \kappa}\right)
\end{aligned}
$$

This is the chiral Gross-Neveu model with $\mathrm{O}\left(2 N_{r}\right) \times$ $\mathrm{O}\left(2 N_{r}\right)$ internal symmetry.

\section{c. class $C I$}

To account for the class CI symmetry $D^{T}=+D$, we note that

$$
\begin{aligned}
& \int d^{3} x f_{A}^{\dagger} D f_{B}=-\int d^{3} x f_{B}^{T} D f_{A}^{*}, \\
& \int d^{3} x f_{B}^{\dagger} D^{\dagger} f_{A}=-\int d^{3} x f_{A}^{T} D^{\dagger} f_{B}^{*},
\end{aligned}
$$

and introduce

$$
\chi_{+}=\frac{1}{\sqrt{2}}\left(\begin{array}{c}
f_{B} \\
-f_{A}^{*}
\end{array}\right), \quad \chi_{-}=\frac{1}{\sqrt{2}}\left(\begin{array}{c}
f_{A} \\
-f_{B}^{*}
\end{array}\right) .
$$


The Lagrangian is then written as

$$
\begin{aligned}
\mathcal{L}_{\mathrm{CI}}= & \chi_{+}^{T} \tau_{y} \otimes D \chi_{+}+\chi_{-}^{T} \tau_{y} \otimes D^{\dagger} \chi_{-} \\
& +\frac{0^{+}}{2}\left[\chi_{+}^{T}\left(-i \tau_{y}\right) \chi_{-}+\chi_{-}^{T}\left(-i \tau_{y}\right) \chi_{+}\right] .
\end{aligned}
$$

At this stage, one notices that $f^{\dagger} \mathcal{H} f$ is invariant under transformations

$$
\chi_{+} \rightarrow O_{+} \chi_{+}, \quad \chi_{-} \rightarrow O_{-} \chi_{-},
$$

where $O_{ \pm}$satisfy

$$
O_{ \pm}^{T} i \tau_{y} O_{ \pm}=i \tau_{y} .
$$

I.e., $O_{ \pm} \in \mathrm{Sp}(1)$. The system is invariant under continuous $\mathrm{Sp}(1) \times \mathrm{Sp}(1)$ rotations. The smearing term breaks this symmetry down to its diagonal subgroup; in order for $O_{ \pm}$to leave the smearing term invariant,

$$
O_{+}^{T} i \tau_{y} O_{-}=i \tau_{y} \Rightarrow \quad O_{+}=O_{-} .
$$

With replicas, the $\mathrm{Sp}(1) \times \mathrm{Sp}(1)$ symmetry is promoted to $\operatorname{Sp}\left(N_{r}\right) \times \operatorname{Sp}\left(N_{r}\right)$ symmetry which is broken down to $\operatorname{Sp}\left(N_{r}\right)$ by the smearing term.

Let us now introduce

$$
\psi^{\dagger}=\left(\chi_{+}^{T}\left(-i \tau_{y}\right), \chi_{-}^{T}\left(-i \tau_{y}\right)\right), \quad \psi=\left(\begin{array}{c}
\chi_{-} \\
\chi_{+}
\end{array}\right) .
$$

They satisfy the "Majorana" condition

$$
\psi^{\dagger}=\psi^{T} \Gamma_{x}\left(-i \tau_{y}\right)
$$

The Lagrangian is written as

$$
\mathcal{L}_{\mathrm{CI}}=\psi^{\dagger}\left(\tau_{0} \otimes i \mathcal{H}_{0}+0^{+}\right) \psi=\sum_{\tau= \pm} \psi_{\tau}^{\dagger}\left(i \mathcal{H}_{0}+0^{+}\right) \psi_{\tau},
$$

where in the second line we made the particle-hole indices explicit, and we have rescaled $0^{+} / 2 \rightarrow 0^{+}$.

Introducing $N_{f}$ flavors and $N_{r}$ replicas and then performing the quench disorder averaging,

$$
\begin{aligned}
\mathcal{L}_{\mathrm{CI}} & =\psi_{a \sigma \iota}^{\dagger}\left(i \mathcal{H}_{0}+0^{+}\right) \delta_{\sigma \tau} \delta_{a b} \psi_{b \tau \iota} \\
& -\frac{g}{2}\left(\psi_{b \tau \iota}^{\dagger} \psi_{a \sigma \iota} \psi_{a \sigma \kappa}^{\dagger} \psi_{b \tau \kappa}-\psi_{b \tau \iota}^{\dagger} c_{z} \psi_{a \sigma \iota} \psi_{a \sigma \kappa}^{\dagger} c_{z} \psi_{b \tau \kappa}\right),
\end{aligned}
$$

where $a, b=1, \ldots, N_{r}, \iota, \kappa=1, \ldots, N_{f}$, and $\sigma, \tau= \pm$.

If we introduce

$$
\bar{\psi}:=\psi^{\dagger} i \Gamma_{z}, \quad \psi^{T}\left(i \tau_{y}\right) \Gamma_{y}=\bar{\psi},
$$

the Lagrangian is then given as

$$
\begin{aligned}
& \mathcal{L}_{\mathrm{CI}}=\bar{\psi}_{a \sigma \iota}\left(\Gamma_{z} \mathcal{H}_{0}-0^{+} i \Gamma_{z}\right) \psi_{a \sigma \iota} \\
& +\frac{g}{2}\left(\bar{\psi}_{a \sigma \iota} \Gamma_{z} \psi_{b \tau \iota} \bar{\psi}_{b \tau \kappa} \Gamma_{z} \psi_{a \sigma \kappa}-\bar{\psi}_{a \sigma \iota} \psi_{b \tau \iota} \bar{\psi}_{b \tau \kappa} \psi_{a \sigma \kappa}\right) .
\end{aligned}
$$

This is the chiral Gross-Neveu model with $\operatorname{Sp}\left(N_{r}\right) \times$ $\operatorname{Sp}\left(N_{r}\right)$ internal symmetry.

\section{2. auxiliary matrix fields}

The four-fermion interactions in the replica field theories can be decoupled by two auxiliary matrix fields which we call $W$ and $V$. Depending on symmetry class, these matrix fields satisfy different constraints.

\section{a. class AIII}

For symmetry class AIII, the fermionic Lagrangian (B11) can be rewritten

$$
\begin{aligned}
\mathcal{L}_{\mathrm{AIII}}= & \bar{\psi}_{a, \iota}\left(\Gamma_{z} \mathcal{H}_{0}-i 0^{+} \Gamma_{z}\right) \delta_{a b} \psi_{b, \iota} \\
& +\bar{\psi}_{a, \iota}\left(W_{a b}+i \Gamma_{z} V_{a b}\right) \psi_{b, \iota} \\
& +\frac{1}{2 g} \operatorname{tr}_{N_{r}}\left(W^{2}+V^{2}\right),
\end{aligned}
$$

where $W$ and $V$ are an $N_{r} \times N_{r}$ hermitian matrix.

\section{b. class DIII}

For symmetry class DIII, the fermionic Lagrangian (B23) can be rewritten

$$
\begin{aligned}
\mathcal{L}_{\text {DIII }}= & \bar{\psi}_{a \iota}\left[\Gamma_{z} \mathcal{H}_{0} \delta_{a b}+0^{+} \Gamma_{z}\left(-i \tau_{y} \otimes I_{N_{r}}\right)_{a b}\right] \psi_{b \iota} \\
& +\bar{\psi}_{a \iota}\left(W_{a b}+i \Gamma_{z} V_{a b}\right) \psi_{b \iota} \\
& +\frac{1}{2 g} \operatorname{tr}_{2 N_{r}}\left(W^{2}+V^{2}\right),
\end{aligned}
$$

where $W$ is a $2 N_{r} \times 2 N_{r}$ real symmetric matrix, while $V$ is a $2 N_{r} \times 2 N_{r}$ pure imaginary antisymmetric matrix.

$$
\text { c. class } C I
$$

To decouple the four fermion terms in the fermionic Lagrangian (B11) for symmetry class CI, we need to introduce two bosonic fields $W_{a \tau, b \tau^{\prime}}$ and $V_{a \tau, b \tau^{\prime}}$ where the first type of index $a, b$ runs over the replica grading, $a, b=1, \cdots, N_{r}$, and the second type of index $\tau, \tau^{\prime}$ runs over the particle-hole grading, $\tau, \tau^{\prime}= \pm$. With these auxiliary fields, the fermionic Lagrangian (B11) can be written as

$$
\begin{aligned}
\mathcal{L}_{\mathrm{CI}}= & \bar{\psi}_{a \sigma \iota}\left(\Gamma_{z} \mathcal{H}_{0}-i 0^{+} \Gamma_{z}\right) \delta_{a b} \delta_{\sigma \tau} \psi_{b \tau \iota} \\
& +\bar{\psi}_{a \sigma \iota}\left(W_{a \sigma, b \tau}+i \Gamma_{z} V_{a \sigma, b \tau}\right) \psi_{b \tau \iota} \\
& +\frac{1}{4 g} \operatorname{tr}_{2}\left(V_{b a} V_{a b}+W_{a b} W_{b a}\right) .
\end{aligned}
$$

Here in the last line, the $2 \mathrm{~d}$ trace is taken over the particle-hole space, and $V_{a b}$ and $W_{a b}$, for the fixed replica indices $a$ and $b$, are viewed as a $2 \times 2$ matrix. They are a real quartenion matrix, i.e., they can be expanded as $V_{a b}=A+B_{x}\left(i \tau_{x}\right)+B_{y}\left(i \tau_{y}\right)+B_{z}\left(i \tau_{z}\right)$ and $W_{a b}=$ 
$C+D_{x}\left(i \tau_{x}\right)+D_{y}\left(i \tau_{y}\right)+D_{z}\left(i \tau_{z}\right)$ with real coefficients $A, B_{x, y, z}, C, D_{x, y, z}$, and also satisfy

$$
\begin{gathered}
W_{b a}=-\left(-i \tau_{y}\right) W_{a b}^{T}\left(i \tau_{y}\right) \quad\left(\equiv-W_{a b}^{R}\right), \\
V_{b a}=+\left(-i \tau_{y}\right) V_{a b}^{T}\left(i \tau_{y}\right) \quad\left(\equiv+V_{a b}^{R}\right) .
\end{gathered}
$$

\section{3. leading order analysis}

To perform the $1 / N_{f}$ expansion, we first compute the propagators of $W$ and $V$ bosons dressed by fermion bubbles. This is depicted diagrammatically in Fig. 1 .

For symmetry class AIII, propagators for $W$ and $V$ bosons are given by

$$
\begin{aligned}
\left\langle W_{a b}(q) W_{c d}\left(-q^{\prime}\right)\right\rangle & =\left\langle V_{a b}(q) V_{c d}\left(-q^{\prime}\right)\right\rangle \\
& =\frac{4}{N_{f} D_{\gamma}} \mathcal{D}(q) \delta_{q, q^{\prime}} \delta_{c b} \delta_{d a},
\end{aligned}
$$

where $\mathcal{D}(q)$ is defined in Eg. (29). Similarly, for class DIII,

$$
\begin{aligned}
\left\langle W_{a b}(-q) W_{c d}\left(q^{\prime}\right)\right\rangle & =\frac{8}{N_{f} D_{\gamma}} \mathcal{D}(q)\left(\delta_{c b} \delta_{d a}+\delta_{c a} \delta_{d b}\right) \delta_{q, q^{\prime}}, \\
\left\langle V_{a b}(-q) V_{c d}\left(q^{\prime}\right)\right\rangle & =\frac{8}{N_{f} D_{\gamma}} \mathcal{D}(q)\left(\delta_{c b} \delta_{d a}-\delta_{c a} \delta_{d b}\right) \delta_{q, q^{\prime}},
\end{aligned}
$$

for $a, b, c, d=1, \ldots, 2 N_{r}$, and for symmetry class CI,

$$
\begin{aligned}
& \left\langle W_{a \sigma, b \tau}(-q) W_{c \sigma^{\prime}, d \tau^{\prime}}\left(q^{\prime}\right)\right\rangle=\frac{8}{N_{f} D_{\gamma}} \mathcal{D}(q) \delta_{q, q^{\prime}} \\
& \times\left(\delta_{c b} \delta_{d a} \delta_{\sigma^{\prime}, \tau} \delta_{\tau^{\prime}, \sigma}-\sigma \tau \delta_{c a} \delta_{d b} \delta_{\sigma^{\prime},-\sigma} \delta_{\tau^{\prime},-\tau}\right), \\
& \left\langle V_{a \sigma, b \tau}(-q) V_{c \sigma^{\prime}, d \tau^{\prime}}\left(q^{\prime}\right)\right\rangle=\frac{8}{N_{f} D_{\gamma}} \mathcal{D}(q) \delta_{q, q^{\prime}} \\
& \times\left(\delta_{c b} \delta_{d a} \delta_{\sigma^{\prime} \tau} \delta_{\tau^{\prime} \sigma}+\sigma \tau \delta_{c a} \delta_{d b} \delta_{\sigma^{\prime},-\sigma} \delta_{\tau^{\prime},-\tau}\right) .
\end{aligned}
$$

\section{Appendix C: non-linear sigma models and topological angle for three-dimensional topological superconductors}

In this appendix, we will derive the non-linear sigma model with topological term for symmetry class AIII. A similar derivation should be possible for symmetry class DIII and CI as well.

We start from the saddle point solution Eq. (67):

$$
(w, v)=(0, v)
$$

where we have set $w=0$ since such saddle points can be reached from $(0, v)$ by continuous $\mathrm{U}\left(N_{r}\right) \times \mathrm{U}\left(N_{r}\right)_{A}$ rotation. I.e., from this saddle point, one can generate other saddle points as $v \gamma^{0} \exp \left(2 i \gamma^{0} \theta\right)$ where $\theta \in \mathrm{u}\left(N_{r}\right)$. In other words, non-zero saddle point $v$ destroys the "axial"
$\mathrm{U}\left(N_{r}\right)_{A}$ symmetry. Such continuous rotation is nothing but the Nambu-Goldstone mode which is the diffuson or Cooperon representing the diffusive motion of fermionic quasiparticles. The coupling of the Nambu-Goldstone mode to fermions are described by

$$
\mathcal{L}=\bar{\psi}\left(\gamma^{k} \partial_{k}+m \gamma^{5}+v \gamma^{0} e^{2 i \gamma^{0} \theta(r)}\right) \psi+\text { const. },
$$

where, with space-dependent $\theta, \exp \left(2 i \gamma^{0} \theta(r)\right)$ represents the Nambu-Goldstone modes, i.e., the fields in the $\mathrm{NL} \sigma \mathrm{M}$,

$$
e^{2 i \gamma^{0} \theta(r)}=\left(\begin{array}{cc}
U & 0 \\
0 & U^{\dagger}
\end{array}\right), \quad U \in \mathrm{U}\left(N_{r}\right)
$$

In the following, we will integrate over fermions to derive the effective action of the Nambu-Goldstone mode. We will do not include the longitudinal fluctuations for a while, but it is important as the integration over them leads to the Gade term.80.81. To prepare for the gradient expansion, we will "unwind" $U$ :

$$
\begin{aligned}
\mathcal{L} & =\bar{\psi}\left(\begin{array}{cc}
m_{0} & \sigma_{k} \partial_{k}+m_{5} \\
U\left(-\sigma_{k} \partial_{k}+m_{5}\right) U^{\dagger} & -m_{0}
\end{array}\right) \psi \\
& =\bar{\psi}\left(\begin{array}{cc}
m_{0} & \sigma_{k} \partial_{k}+m_{5} \\
-\sigma_{k} \partial_{k}+m_{5}-\sigma_{k} U \partial_{k} U^{\dagger} & -m_{0}
\end{array}\right) \psi,
\end{aligned}
$$

where we have renamed $v \rightarrow m_{0}$ and $m \rightarrow m_{5}$. Thus, our action can be written as

$$
\begin{gathered}
\mathcal{L}=\bar{\psi}\left(\gamma^{k} \partial_{k}+m_{0} \gamma^{0}+m_{5} \gamma_{5}+\gamma^{k} A_{k}+\gamma^{0} \gamma^{k} B_{k}\right) \psi \\
\text { where } \quad A_{k}=-B_{k}=(1 / 2) U \partial_{k} U^{\dagger} .
\end{gathered}
$$

The effective action is obtained by integrating over fermions,

$$
\int \mathcal{D}[\bar{\psi}, \psi] e^{-\int d^{3} x \mathcal{L}}=e^{-S_{\mathrm{eff}}[U]}
$$

and it can subsequently be expanded in powers of $A_{i}, B_{i}$ and their gradients as

$$
\begin{aligned}
S_{\text {eff }} & =-\operatorname{Tr}\left(G_{0}^{-1}-V\right) \\
& =-\operatorname{Tr} \ln G_{0}^{-1}+\sum_{n=1}^{\infty} \frac{1}{n} \operatorname{Tr}\left(G_{0} V\right)^{n},
\end{aligned}
$$

where $V=\gamma^{i} A_{i}+\gamma^{0} \gamma^{i} B_{i}$, and the free propagator is given by

$$
G_{0}(k)=-\frac{\gamma^{k} i k_{k}+m_{0} \gamma^{0}+m_{5} \gamma^{5}}{k^{2}+m_{0}^{2}+m_{5}^{2}}
$$

with $M^{2}=m_{0}^{2}+m_{5}^{2}$.

To the $3 r d$ order in the gradient expansion, the effective 
action is $\left[S_{\text {eff }}^{(n)}=(1 / n) \operatorname{Tr}\left(G_{0} V\right)^{n}\right]$

$$
\begin{aligned}
S_{\mathrm{eff}}^{(2)}= & \int d^{3} x\left[\frac{\Lambda_{\mathrm{Uv}}}{3 \pi^{2}} \operatorname{tr}\left(A_{j} A_{j}+B_{j} B_{j}\right)\right. \\
& \left.-\frac{i m_{5}}{2 \pi|M|} \epsilon^{i j k} \operatorname{tr}\left(A_{i} \partial_{j} B_{k}\right)+\frac{m_{0}^{2}}{8 \pi|M|} \operatorname{tr}\left(\partial_{j} U^{\dagger} \partial_{j} U\right)\right], \\
S_{\mathrm{eff}}^{(3)}= & \int d^{3} x\left[-\frac{i m_{5}^{3}}{6 \pi|M|^{3}} \epsilon^{i j k} \operatorname{tr}\left(B_{i} B_{j} B_{k}\right)\right. \\
& \left.-\frac{i m_{5}\left(m_{0}^{2}+2 m_{5}^{2}\right)}{4 \pi|M|^{3}} \epsilon^{i j k} \operatorname{tr}\left(A_{i} A_{j} B_{k}\right)\right] .
\end{aligned}
$$

By noting that

$$
\int d^{3} x \epsilon^{i j k} \operatorname{tr} A_{i} \partial_{j} B_{k}=-2 \int d^{3} x \epsilon^{i j k} \operatorname{tr} B_{i} B_{j} B_{k},
$$

the combined 2 nd and 3rd order terms can be written as

$$
\begin{aligned}
S_{\mathrm{eff}}^{(2)}+S_{\mathrm{eff}}^{(3)}= & +\frac{\Lambda_{\mathrm{UV}}}{3 \pi^{2}} \int d^{3} x \operatorname{tr}\left(A_{j} A_{j}+B_{j} B_{j}\right) \\
& +\frac{m_{0}^{2}}{8 \pi|M|} \int d^{3} x \operatorname{tr}\left(\partial_{j} U^{\dagger} \partial_{j} U\right) \\
& -\frac{1}{8|M|^{3}}\left[9 m_{5} m_{0}^{2}+4 m_{5}^{3}\right] 2 \pi i \Gamma[U]
\end{aligned}
$$

Observe that when $m_{0}=0$, if we introduce new gauge fields $A^{ \pm}$by

$$
A=\frac{1}{2}\left(A^{+}+A^{-}\right), \quad B=\frac{1}{2}\left(A^{+}-A^{-}\right),
$$

the effective action can be written as the double Chern-
Simons theory:

$$
\begin{aligned}
S_{\mathrm{eff}}= & +\frac{\Lambda_{\mathrm{UV}}}{3 \pi^{2}} \int d^{3} x \operatorname{tr}\left(A_{j} A_{j}+B_{j} B_{j}\right) \\
& -\frac{1}{2} \frac{m_{5}}{\left|m_{5}\right|} \frac{i}{4 \pi} \sum_{\sigma= \pm} \sigma \int d^{3} x \epsilon^{i j k} \\
& \times \operatorname{tr}\left(A_{i}^{\sigma} \partial_{j} A_{k}^{\sigma}+\frac{2}{3} A_{i}^{\sigma} A_{j}^{\sigma} A_{k}^{\sigma}\right) .
\end{aligned}
$$

The effective action (C11) [and (C14) when $m_{0}=0$ ] that we have derived should be regularized as signaled by the appearance of the term linear in $\Lambda_{\mathrm{UV}}$ in (C11) and (C14). We introduce the regularized action $S_{\text {eff }}^{(R)}$ by the Pauli-Villars regularization with the regulator mass $m_{R}$ as

$$
S_{\mathrm{eff}}^{(R)}=S_{\mathrm{eff}}\left(m_{5}, m_{0}\right)-\lim _{\left|m_{R}\right| \rightarrow \infty} S_{\mathrm{eff}}\left(m_{R}, 0\right) .
$$

We choose the sign of the regulator mass as

$$
\operatorname{sgn}\left(m_{R}\right)=-\operatorname{sgn}\left(m_{5}\right)
$$

in which case $m_{5}>0$ is a topological/non-topological phase. We then conclude

$$
\begin{aligned}
& S_{\mathrm{eff}}^{(R)}=\frac{m_{0}^{2}}{8 \pi|M|} \int d^{3} x \operatorname{tr}\left(\partial_{j} U^{\dagger} \partial_{j} U\right) \\
& \quad+\left[\frac{-1}{8|M|^{3}}\left(9 m_{5} m_{0}^{2}+4 m_{5}^{3}\right)+\frac{m_{R}^{3}}{2\left|m_{R}\right|^{3}}\right] 2 \pi i \Gamma[U] .
\end{aligned}
$$

(2009).

14 C. Brüne, C. X. Liu, E. G. Novik, E. M. Hankiewicz, H. Buhmann, Y. L. Chen, X. L. Qi, Z. X. Shen, S. C. Zhang, and L. W. Molenkamp, Phys. Rev. Lett. 106, 126803 (2011).

15 Andreas P. Schnyder, Shinsei Ryu, Andreas W. W. Ludwig, Phys. Rev. Lett. 102, 196804 (2009).

16 Pavan Hosur, Shinsei Ryu, Ashvin Vishwanath, Phys. Rev. B 81, 045120 (2010).

17 Ryuichi Shindou, Shuichi Murakami, Phys. Rev. B 79, 045321 (2009).

18 Ryuichi Shindou, Ryota Nakai, Shuichi Murakami, New Journal of Physics, 12, 065008 (2010).

19 H.-M. Guo, G. Rosenberg, G. Refael, M. Franz, Phys. Rev. Lett. 105, 216601 (2010).

20 Pallab Goswami and Sudip Chakravarty arXiv:1101.2210.

21 A. M. M. Pruisken, Nucl. Phys. B 235 277, (1984).

22 H. Levine, S. Libby, and A. M. M. Pruisken, Phys. Rev. Lett. 51 20, (1983).

23 H. Levine, S. Libby, and A. M. M. Pruisken, Nucl. Phys. B 240 [FS12] 30, (1983); ibid., Nucl. Phys. B 240 [FS12] 49, (1983); ibid., Nucl. Phys. B 240 [FS12] 71, (1983).

24 K. G. Wilson, Phys. Rev. D 10, 2445 (1974).

25 X.-L. Qi, T.-L. Hughes, and S.-C. Zhang, Phys. Rev. B 78, 
195424 (2008).

26 See, for example, A. W. W. Ludwig, M. P. A. Fisher, R. Shankar, G. Grinstein, Phys. Rev. B 50, 7526 (1994); S. Ryu, C. Mudry, A. Furusaki, and A. W. W. Ludwig, Phys. Rev. B 75, 205344 (2007), and references therein.

27 A. MacKinnon and B. Kramer, Z. Phys. B 53, 1 (1983).

28 M. Onoda, Y. Avishai, and N. Nagaosa, Phys. Rev. Lett. 98, 076802 (2007).

29 H. Obuse, A. Furusaki, S. Ryu, and C. Mudry, Phys. Rev. B 76, 076802 (2007); Shinsei Ryu, Christopher Mudry, Hideaki Obuse, and Akira Furusaki, New J. Phys. 12, 065005 (2010).

30 A. Yamakage, K. Nomura, K.-I. Imura, and Y. Kuramoto, J. Phys. Soc. Jpn. 80, 053703 (2011).

31 J. Li, R.-L. Chu, J. K. Jain, and S.-Q. Shen, Phys. Rev. Lett. 102, 136806 (2009).

32 H. Jiang, L. Wang, Q.-F. Sun, and X. C. Xie, Phys. Rev. B 80, 165316 (2009).

33 C. W. Groth, M. Wimmer, A. R. Akhmerov, J. Tworzydlo, and C. W. J. Beenakker, Phys. Rev. Lett. 103, 196805 (2009).

34 E. Prodan, J. Phys. A: Math. Theor. 44, 113001 (2011).

35 D. J. Gross, and A. Neveu Phys. Rev. D 10, 3235 (1974).

36 Simon Hands, Aleksandar Kocic, and John B. Kogut, Annals of Physics 224, 29 (1993).

37 B. Rosenstein, B. J. Warr, and S. H. Park, Phys. Rep. 205, 59 (1991).

38 J. Zinn-Justin, Quantum Field Theory and Critical Phenomena, (Oxford University Press, New York, 1993).

39 Moshe Moshe and Jean Zinn-Justin, Phys. Rept. 385, 69 (2003).

40 Yoichi Asada, Keith Slevin, and Tomi Ohtsuki, J. Phys. Soc. Jpn. 74 Suppl. 238 (2005).

41 D. Belitz and T. R. Kirkpatrick, Rev. Mod. Phys. 66, 261 (1994)

42 Matthew S. Foster, Andreas W. W. Ludwig, Phys. Rev. B 77, 165108 (2008)

43 Qinghong Cui, Xin Wan, Kun Yang, Phys. Rev. B 70, 094506 (2004).

${ }^{44}$ Class AIII also appears in an effective description of QCD; J. J. M. Verbaarschot and I. Zahed, Phys. Rev. Lett. 70, 3853 (1993); J. J. M. Verbaarschot, Phys. Rev. Lett. 72, 2531 (1994)

45 Rahul Roy, arXiv:0803.2868.

46 Xiao-Liang Qi, Taylor L. Hughes, Srinivas Raghu, and Shou-Cheng Zhang, Phys. Rev. Lett. 102, 187001 (2009).

47 M. M. Salomaa and G. E. Volovik, Phys. Rev. B 37, 9298 (1988).

48 S. Murakawa et al., Phys. Rev. Lett. 103, 155301 (2009).

49 S. Murakawa, Y. Wada, Y. Tamura, M. Wasai, M. Saitoh, Y. Aoki, R. Nomura, Y. Okuda, Y. Nagato, M. Yamamoto, S. Higashitani, K. Nagai, J. Phys. Soc. Jpn. 80, 013602 (2011).

50 Andreas P. Schnyder and Shinsei Ryu Phys. Rev. B 84, 060504(R) (2011)

51 Shinsei Ryu, Phys. Rev. B 79, 075124 (2009).

52 Y. Nambu and G. Jona-Lasinio, Phys. Rev. 122345, (1961).

53 R. Gade and F. Wegner, Nucl. Phys. B 360, 213 (1991); R. Gade, ibid. 398, 499 (1993).

54 Antonio M. Garcia-Garcia and Emilio Cuevas, Phys. Rev. B 74, 113101 (2006).

55 Paul Fendley, in New Theoretical Approaches to
Strongly Correlated Systems, edited by A. M. Tsvelik (Kluwer Academic Publishers, The Netherlands, 2001); arXiv: cond-mat/0006360; P. Fendley, Phys. Rev. B 63, 104429 (2001).

56 A. G. Abanov, P. B. Wiegmann, Nucl. Phys. B, 570, 685.

57 T. Senthil, Matthew P. A. Fisher, Leon Balents, and Chetan Nayak, Phys. Rev. Lett. 81, 4704 (1998).

58 T. Senthil and Matthew P. A. Fisher, Phys. Rev. B 61, 9690 (2000); N. Read and Andreas W. W. Ludwig, Phys. Rev. B 63, 024404 (2000); J. T. Chalker, N. Read, V. Kagalovsky, B. Horovitz, Y. Avishai, and A. W. W. Ludwig, Phys. Rev. B 65, 012506 (2001).

59 T. Senthil, J. B. Marston, and M. P. A. Fisher, Phys. Rev. B 60, 4245 (1999); Ilya A. Gruzberg, Andreas W. W. Ludwig, and N. Read, Phys. Rev. Lett. 82, 4524 (1999).

60 For a similar discussion for the $\mathrm{O}(N)$ non-linear sigma model in $(2+1)$ dimensions, see Pouyan Ghaemi and Shinsei Ryu, arXiv:1012.5840.

61 One should be warned that NL $\sigma$ Ms might not be useful in three dimensions, since they are not perturbatively renormalizable. On the other hand, it can be renormalizable non-perturbatively (i.e., in the Wilsonian sense). (They are also renormalizable in the large- $N$ expansion, although in the context of Anderson localization, $N$ in NL $\sigma \mathrm{Ms}$ is proportional to the number of replicas $N_{r}$ and should be taken to be zero at end of the calculations.)

62 D. J. Thouless, M. Kohmoto, M. P. Nightingale, and M. den Nijs, Phys. Rev. Lett. 49, 405 (1982)

${ }^{63}$ K. Nomura, M. Koshino, and S. Ryu, Phys. Rev. Lett. 99, 146806 (2007).

64 S. Ryu, C. Mudry, H. Obuse, and A. Furusaki, Phys. Rev. Lett. 99, 116601 (2007).

65 P. M. Ostrovsky, I. V. Gornyi and A. D. Mirlin, Phys. Rev. Lett. 98, 256801 (2007).

66 M. Barkeshli and X.-L. Qi (2011), arxiv:1101.3104.

67 Doron L. Bergman, arXiv: 1101.4233.

68 J. V. Porto and J. M. Parpia, Phys. Rev. Lett. 74, 4667 (1995).

69 W. P. Halperin et al, Physica B 329-333, 288 (2003).

${ }^{70}$ Y. S. Hor, A. J. Williams, J. G. Checkelsky, P. Roushan, J. Seo, Q. Xu, H. W. Zandbergen, A. Yzdani, N. P. Ong, and R. J. Cava, Phys. Rev. Lett. 104, 057001 (2010).

71 L. Wray, S. Xu, J. Xiong, Y. Xia, D. Qian, H. Lin, A. Bansil, Y. Hor, R. J. Cava, M. Z. Hasan, Nature Phys. 6 855 (2010).

72 L. Fu and E. Berg, Phys. Rev. Lett. 105, 097001 (2010).

73 Satoshi Sasaki, M. Kriener, Kouji Segawa, Keiji Yada, Yukio Tanaka, Masatoshi Sato, Yoichi Ando, arXiv: 1108.1101.

74 S. Ryu, J. E. Moore, and A. W. W. Ludwig, arxiv: 1010.0936

75 Z. Wang, X. -L. Qi, and S. -C. Zhang, Phys. Rev. B 84, 014527 (2011)

76 Y. Onose, T. Ideue, H. Katsura, Y. Shiomi, N. Nagaosa, and Y. Tokura, Science 329, 297 (2010).

77 Hideaki Obuse, Akira Furusaki, Shinsei Ryu, and Christopher Mudry, Phys. Rev. B 78, 115301 (2008).

78 Xiangang Wan, Ari Turner, Ashvin Vishwanath, Sergey Y. Savrasov, Phys. Rev. B 83, 205101 (2011).

79 G. E. Volovik, Pisma Zh.Eksp.Teor.Fiz. 93:69-72,2011; JETP Lett.93:66-69,2011

80 A. Altland and B. D. Simons, Nucl. Phys. B 562, 445 (1999).

81 A. Altland, R. Merkt, Nucl. Phys. B 607, 511 (2001). 